\title{
A Comparative Code Study for Quantum Fault Tolerance
}

\author{
Andrew Cross * $\quad$ David P. DiVincenzo ${ }^{\dagger} \quad$ Barbara M. Terhal $^{\ddagger}$
}

February 6, 2009

\begin{abstract}
We study a comprehensive list of quantum codes as candidates for codes used at the physical level in a fault-tolerant code architecture. Using the Aliferis-Gottesman-Preskill (AGP) ex-Rec method we calculate the pseudo-threshold for these codes against depolarizing noise at various levels of overhead. We estimate the logical noise rate as a function of overhead at a physical error rate of $p_{0}=1 \times 10^{-4}$. The Bacon-Shor codes and the Golay code are the best performers in our study.
\end{abstract}

\section{Contents}

1 Introduction

1.1 The Code Architecture . . . . . . . . . . . . . . . . . . . 3

2 Preliminaries 4

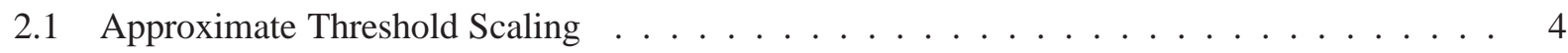

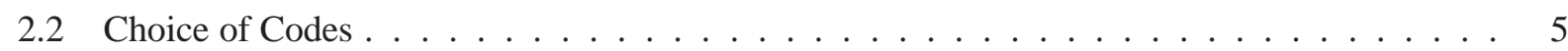

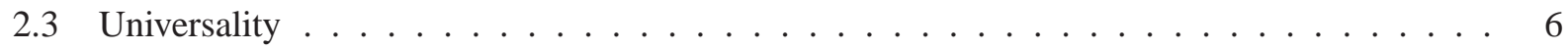

3 Error Correction Circuits

3.1 Specific Code Considerations . . . . . . . . . . . . . . . . 8

4 The Aliferis-Gottesman-Preskill (AGP) Method 10

4.1 Monte-Carlo Implementation of Method . . . . . . . . . . . . . . . . . . . . 12

4.2 Software and Computer Use . . . . . . . . . . . . . . . . . . 13

5 Results 13

5.1 Perfect Ancillas . . . . . . . . . . . . . . . . . . . 14

5.2 Pseudo-Thresholds . . . . . . . . . . . . . . . . . . . 15

5.3 Influence of Storage Errors . . . . . . . . . . . . . . . . . . 16

5.4 Logical Error Rate versus Overhead . . . . . . . . . . . . . . . . . 16

${ }^{*}$ Department of Electrical Engineering and Computer Science, MIT, Cambridge, MA, 02139, USA and IBM Watson Research Center, P.O. Box 218, Yorktown Heights, NY, USA 10598. andrew . w . cross @ gmail . com

${ }^{\dagger}$ IBM Watson Research Center, P.O. Box 218, Yorktown Heights, NY, USA divince@wat son . ibm. com

${ }^{\ddagger}$ IBM Watson Research Center, P.O. Box 218, Yorktown Heights, NY, USA 10598. bterhal@gmail . com 
A Various Aspects of Steane-EC 21

A.1 Latin Rectangle Method for Optimizing Encoding Circuits . . . . . . . . . . . . . . . 22

B Syndrome Decoding

C Data Tables

\section{Introduction}

A great insight in the early history of quantum computing was that almost perfectly reliable quantum computation is possible with physical devices subjected to noise as long as the noise level is not too large. This observation has given us confidence that, ultimately, it will be possible to build a functioning quantum computer. However, the "threshold theorem" that indicates how much noise can be tolerated has not otherwise given a very optimistic prognosis for progress. For example, the well-studied seven qubit Steane code $[[7,1,3]]$ has a threshold against adversarial noise that is in the $O\left(10^{-5}\right)$ range. This level of noise is far lower than anything that has been achieved in any laboratory - it is actually a significantly lower error rate than many experiments are even capable of measuring.

In this paper we analyze both previously considered and new additional quantum codes and determine their thresholds, logical error rates and overheads using the ex-Rec method developed in [1]. This allows us for the first time to compare the relative merit of many schemes. We will argue how the studied codes could serve as bottom (physical-level) codes in a fault-tolerant code architecture that minimizes the overall coding overhead.

In order to carry out this comparative code study we must make some simplifying assumptions. First of all, we assume that gates can be performed in a non-local geometry. It is likely that an ultimate quantum architecture will be largely restricted to local 1D, 2D or 3D geometries, hence the threshold numbers that we estimate will be affected by this architecture constraint. As was shown in [2, 3], the price for locality may be modest for small codes, but it will typically be worse for larger codes [2] since a bigger effort will have to be mounted to make the error-correction circuits local.

Secondly, the noise model for our study is a simple depolarizing noise model with equal probabilities for Pauli $X, Y$ and $Z$ errors. In particular, we assume that any single qubit location in the quantum circuit undergoes a Pauli $X, Y$ or $Z$ error with equal probability $p_{0} / 3$. A two-qubit gate undergoes no error with probability $1-p_{0}$ and one of 15 errors with probability $p_{0} / 15$. The reason for choosing this model is that it is the simplest unbiased choice for a noise model given that a comparative study of the performance of codes for general noise models (such as superoperator noise) is infeasible computationally. We do not expect the performance of these codes to differ greatly if we would choose a biased depolarizing noise model, since there are no intrinsic biases in the codes themselves. To deal with biased noise, it may be more advantageous to use specific biased code constructions such a non-square Bacon-Shor or surface code or the use of the repetition code against high-rate dephasing noise in [4].

We do not establish rigorous threshold lower-bounds by counting the number of malignant sets of faults as in [1]. Instead of counting or sampling of malignant faults, we simply simulate the depolarizing noise and keep track of when it leads to failures. We put error bars on our results such that a rigorous lower bound on the pseudo-threshold is within this statistical error bar. The level-1 pseudo-threshold is the value of $p_{0}$ 
where the level-1 encoded error rate $p_{1}=p_{0}[6]$. It is only the pseudo-threshold that is of interest in this study of bottom codes since we envision, see Section 1.1, that a different code would be used in the next level of encoding.

Our study is in some sense a continuation of the first comparative code study by Steane [5]. Our analysis goes beyond these previous Monte Carlo studies of quantum fault-tolerance in that it includes more codes and focuses on a fault-tolerance analysis of the logical CNOT gate. One of the problems with comparing threshold estimates in the literature is that threshold numbers for different codes have been obtained by different methods, some more rigorous than other's. We believe that it would be advantageous to stick to one clear, rigorously motivated method. The AGP method has the advantage of being tied to a fully rigorous analysis [1] and the AGP method when used to (approximately) count the number of malignant sets of faults can give tight estimates of the (pseudo)-threshold.

With a few exceptions, we use standard Steane error-correction circuits (and sometimes omit possible code-specific optimizations) that allows us to compare codes directly. We discuss these possible code optimizations and our choices in Section 2 . We do not separately analyze Knill's post-selected and Fibonacci schemes [9, 10] but we plot Knill's numbers in Figure 14. One scheme that is not included is the surface code scheme described in Ref. [11]. However, we do study surface codes in the original setting of [12] using Shor error-correction circuits.

\subsection{The Code Architecture}

The usefulness of error correction in computation is roughly measured by two parameters. First is the reduction in error rate that is obtained by using the code; this feature depends on the (pseudo)-threshold of the code for the particular noise model and the error correction circuits that are used. The second figure of merit is the smallness of the overhead that is incurred by coding. There is a tradeoff that one can expect between overhead and logical error-rate that mimics the trade-off between distance and rate of error-correction codes, see Figure 14. Since error levels of physical implementations are expected to be high, optimistically in the range from $O\left(10^{-2}\right)$ to $O\left(10^{-6}\right)$, it is clear that at the bottom level, optimizing the threshold has priority over optimizing overhead. This leads us to consider the following simple code architecture. At the physical level, we use a bottom code $C_{\text {bot }}$ which is chosen to have a high noise threshold and a reasonable overhead. This paper will be devoted to a comparative study of such codes. We will pick some illustrative numbers to argue how one can envision completing the code architecture. We will see that one can find a bottom code that maps a base error rate of $p_{0}=O\left(10^{-4}\right)$ onto a logical error rate of $p_{1}=O\left(10^{-7}\right)$ (see Section 5). To run a reasonable-sized factoring algorithm one may need an logical error rate of, say, $O\left(10^{-15}\right) 1$. Thus one needs a top code $C_{\text {top }}$ that brings the error rate $O\left(10^{-7}\right)$ to $O\left(10^{-15}\right)$. The desirable features of the top code are roughly as follows (see also the Discussion at the end of the paper). The top code is a block code $[[n, k, d]]$ with good rate $k / n$ in order to minimize the overhead. The improvement in error-rate for a code which can correct $t$ errors is roughly

$$
p_{1} \approx p_{0}\left(\frac{p_{0}}{p_{\text {th }}}\right)^{t}
$$

where $p_{0}$ is the unencoded error-rate and $p_{\text {th }}$ is the threshold error rate. Thus in order to get from $p_{0}=$ $O\left(10^{-7}\right)$ to $p_{1}=O\left(10^{-15}\right)$ we could use a code which can correct 4 errors and has a threshold of $O\left(10^{-5}\right)$. In [13] Steane studied several block-codes which may meet these demands. The polynomial codes would also be an interesting family to study in this respect.

\footnotetext{
${ }^{1}$ An $n$-bit number can be factored using a circuit with space-time complexity of roughly $360 n^{4}$ [20], so RSA-1024 could be broken using a circuit with $O\left(10^{15}\right)$ potential fault locations. Using different architectures, it may be possible to reduce this to $O\left(10^{11}\right)$ see for example [21].
} 


\section{Preliminaries}

For our study it is necessary to select a subset of quantum codes. We focus on codes that are likely to have a good threshold, possibly at the cost of a sizeable but not gigantic overhead. To first approximation the threshold is determined by the equation

$$
p_{\mathrm{th}}=N p_{\mathrm{th}}^{\mathrm{t+1}} \Rightarrow p_{\mathrm{th}}=N^{-1 / t},
$$

where $t$ is the number of errors that the code can correct and $N$ is a combinatorial factor counting the sets of $t+1$ locations in an encoded gate that lead to the encoded gate failing. It is clearly desirable to minimize the number of locations and maximize $t$. This consideration has led us to primarily consider CalderbankShor-Steane (CSS) codes. Any stabilizer quantum code is CSS if and only if the CNOT gate is a transversal gate [17]. The advantage of a transversal CNOT is that it minimizes the size of the encoded CNOT; the bulk of the CNOT rectangle will be taken up by error correction. This is favorable for the noise threshold of $C_{\text {bot }}$. Secondly, minimizing the error-rate of the encoded gate $C_{\text {bot }}(\mathrm{CNOT})$ will be useful at the next level of encoding, because CNOTs occur frequently in EC and their error rates play a large role in determining whether error rates are below the threshold (of $C_{\text {top }}$ ). However to demonstrate that this restriction to CSS codes is warranted we also consider the non-CSS 5-qubit code $[[5,1,3]]$ which is the smallest code that can correct a single error. We indeed find that this code performs worse than Steane's 7-qubit code $[[7,1,3]]$, see Section 5 and the Data Tables in Appendix $\mathrm{C}$

\subsection{Approximate Threshold Scaling}

In this section we discuss the global behavior of the noise threshold as a function of block size $n$, distance, and other code properties. Let us consider Eq. (2) and see how we can get the best possible threshold. An upper-bound on $N$ is $\left(\begin{array}{c}A \\ t+1\end{array}\right)$ where $A$ is the total number of locations in the encoded gate (rectangle). Ideally, a code family has a distance that is linear in $n$, i.e. $t$ is linear in $n$. Let us assume for simplicity that only some fraction of all locations appears in the malignant fault sets of size $t+1$, i.e. we model $N \approx\left(\begin{array}{c}A_{\text {mal }} \\ t+1\end{array}\right)$ where $A_{\text {mal }}<A$. The locations in $A_{\text {mal }}$ are in some sense the weak spots in the circuits; overall failure is most sensitive to failure at these locations. $A_{\text {mal }}$ may be either linear or super-linear in the block size $n$. In case $A_{\text {mal }}$ scales linearly with $n$, and $t=\delta n$ for some $\delta \leq 1 / 4$, the threshold in Eq. (2) increases as a function of $n$ and asymptotes in the limit of large $n$ to a finite value. Indeed, for $A_{\text {mal }}=\alpha n$ and $\delta \ll \alpha$ (which is typically the case since $t \leq n / 4$ ) we get, using Sterling's approximation,

$$
p_{\text {th }}=\lim _{n \rightarrow \infty}\left(\begin{array}{c}
\alpha n \\
\delta n+1
\end{array}\right)^{-1 /(\delta n)}=\frac{\delta}{e \alpha}+O\left(\frac{\delta^{2}}{\alpha^{2}}\right)
$$

Such monotonic increase of the threshold with block-size is clearly desirable. It is also clear that when $t$ is constant, for any polynomial $A_{\text {mal }}=\operatorname{poly}(n)$, the threshold $p_{\text {th }}$ in Eq. (2) decreases as a function of $n$. When $A_{\text {mal }}$ scales super-linearly with $n$ and $t$ is linear in $n$ we get the following behavior. First, the threshold increases with $n$ (the effect of larger $t$ ), then the threshold declines since the effect of a super-linear $A_{\text {mal }}$ starts to dominate. For codes and EC circuits with this behavior, it is thus of interest to determine where this peak threshold performance occurs. We will see some evidence of these peaks in Figure 10 in Section 5 .

Now let us consider the scaling of $A$ (and $A_{\text {mal }}$ ) in case we use Steane error correction. In Appendix $\mathrm{A}$ we review how we can bound $A$ for a CSS code with Steane error correction, but a rough estimate is that

$$
A=c_{1} A_{\mathrm{enc}}+c_{2} A_{\mathrm{ver}}+c_{3} n .
$$

Here $A_{\text {enc }}$ is the number of locations in the encoding of the ancillas for error correction, and $A_{\text {ver }}$ is the number of locations in the verification of the ancillas for error correction. The additional term linear in $n$ 
comes from the transversal encoded gates and the transversal syndrome extractions. For a CSS code and the standard encoding construction (see Appendix $\mathrm{A}$ ), $A_{\text {enc }}$ typically scales as $O(w n)$ where $w$ is the maximum Hamming weight of the rows of the generator matrix of either $C_{1}$ or $C_{2}^{\perp}$ in standard form. However this standard construction may be sub-optimal, since by bringing the generator matrix in standard form one can increase the maximum weight of its rows.

For Steane-EC the full verification of the ancilla block requires other ancillas blocks; a fully fault-tolerant verification would give a pessimistic scaling of $A_{\text {ver }}=O(w n t)$. However it is not necessarily desirable to have strict fault tolerance as long as the total probability of low-weight faults that produce faults with weight $t+1$ or more is low, see the discussion in Section 4 On the other hand for increasing $n$ the number of verification rounds should at least be increasing with $n$, perhaps $O(\log n)$ would be sufficient. If we assume that $A_{\text {mal }}$ scales similarly as $A$, it follows that if we look for linear-scaling $A_{\text {mal }}$ we need to look at code families which have simple encoders, scaling linearly with $n$. This seems only possible for stabilizer codes with constant weight stabilizers, such as quantum LDPC codes [18] and surface codes or for the Bacon-Shor codes (which has encoders that use $O(n)$ 2-qubit gates).

For the Bacon-Shor and surface codes the distance $t$ does not scale linearly with $n$ (but as $\sqrt{n}$ ). Nonetheless, the work in [12] shows that the effective distance for the surface codes does scale linearly with the block size, since there are very few incorrectible errors of weight $O(t)$. For the Bacon-Shor code family, where one has less syndrome information, this behavior has not been observed [19] (see also Figure 9).

For code families with constant-weight stabilizers an interesting alternative to Steane-EC [33] is the use of Shor-EC [43] where the syndrome corresponding to each stabilizer is extracted using a cat state or simple unencoded qubit ancillas. As for ancilla verification in Steane-EC, the syndrome extraction needs to be repeated to make the circuits more fault-tolerant. In Section 5 we will see the effect on the threshold of using Steane-EC versus Shor-EC for the surface codes [28, 29], see Figure 11 of Section 5] It is striking that the surface codes with Shor EC are the only known examples of a code family with a finite $n \rightarrow \infty$ threshold. This is despite the $O(n \sqrt{n})$ scaling of the total number of locations $A$ of the Shor error correction circuit.

\subsection{Choice of Codes}

The codes that we have studied are listed in Table 1 All codes in this table are CSS codes with the exception of the [[5,1,3]] code. Some of these codes have been previously analyzed by Steane in Ref. [5]. There exist various families of binary CSS quantum codes; the families are the quantum Reed-Muller codes, the quantum Hamming codes, the quantum BCH codes, the surface codes and the sub-system Bacon-Shor codes. In our study we consider only a single member of the quantum Reed-Muller family, a $[[15,1,3]]$ code, since these codes typically don't have very good distance versus block-size [22]. The $[[15,1,3]]$ code was first constructed in [23] from a punctured Reed-Muller code $\operatorname{RM}(1,4)$ and its even sub-code. It is the smallest known distance- 3 code with a transversal $\mathrm{T}$ gate.

We study various quantum Hamming and quantum BCH codes (see a complete list of quantum $\mathrm{BCH}$ codes of small block-size in [24]) which are constructed from self-orthogonal classical Hamming and $\mathrm{BCH}$ codes respectively. We have chosen those codes that encode a single qubit and have maximum distance for a given block size. We have included the previously studied Bacon-Shor codes and the surface codes in our study. We have also included the concatenated 7-qubit code $[[49,1,9]]$ which we use in the way that was proposed by Reichardt in [25], see the details in Section 3.1]

Another family of codes that has been proposed for fault tolerance [26] are the quantum Reed-Solomon codes or polynomial codes. These are codes that are naturally defined on qudits. In this study we consider them as candidates for bottom codes. An alternative use is to consider them as top codes where one uses a bottom code to map the qubits onto qudits. In our study we assume that quantum information is presented 


\begin{tabular}{|c|c|}
\hline PARAMETERS & NOTES \\
\hline$[[5,1,3]]$ & non-CSS five qubit code [27] \\
\hline$[[7,1,3]]$ & Steane's 7-qubit code (doubly-even dual-containing) [8] \\
\hline$[[9,1,3]],[[25,1,5]],[[49,1,7]],[[81,1,9]]$ & Bacon-Shor codes [19] \\
\hline$[[15,1,3]]$ & Quantum Reed-Muller code [22, 23] \\
\hline$[[13,1,3]],[[41,1,5]],[[85,1,7]]$ & Surface codes [28, 29] \\
\hline$[[21,3,5]]$ & Dual-containing polynomial code on $G F\left(2^{3}\right)[7$ \\
\hline$[[23,1,7]]$ & Doubly-even dual-containing Golay code (cyclic) [34] \\
\hline$[[47,1,11]]$ & Doubly-even dual-containing quadratic-residue code (cyclic) [24] \\
\hline$[[49,1,9]]$ & Concatenated $[[7,1,3]]$ Hamming code [25] \\
\hline$[[60,4,10]]$ & Dual-containing polynomial code on $G F\left(2^{4}\right)$ [7] \\
\hline$[[79,1,15]],[[89,1,17]],[[103,1,19]],[[127,1,19]]$ & BCH codes, not analyzed [24] \\
\hline
\end{tabular}

Table 1: A list of the codes included in our study.

in the form of qubits and hence we will consider these codes as binary stabilizer codes. We specifically chose to include the $[[21,3,5]]$ (a concatenated $[[7,1,4]]_{8}$ ) and the $[[60,4,10]]$ (a concatenated $[[15,1,8]]_{16}$ ) code from the family of dual-containing polynomial codes over $G F\left(2^{m}\right)$, because they are the smallest error-correcting polynomial codes in this family.

We find it impractical to simulate the encoded CNOT gate for BCH codes in this table which have blocksize larger than $[[47,1,11]]$, see 4.2. The threshold for these bigger codes will benefit considerably from the fact that $t / n$ is quite high. Some semi-analytical values for the thresholds of these codes have been given in [5]. Even with good thresholds, these bigger $\mathrm{BCH}$ codes have limited applicability due to their large overhead. The bottom code should be picked to obtain a logical error rate that is well below the threshold of some good block code but only at the price of a moderate overhead.

\subsection{Universality}

Universality for CSS codes can in principle be obtained using the technique of injection-and-distillation [10, 14, 15]. Let us briefly review how one may perform fault-tolerant computation for CSS codes for which, of the Clifford group gates, only the CNOT and Pauli operations are transversal. If one is able to perform any Clifford group gate transversally, including $\mathrm{H}$ and $\mathrm{S}$, it is well known how to obtain a universal set of gates [30]. Note that a CSS code with only its transversal CNOT gives us the ability to fault-tolerantly prepare the states $\{|\overline{+}\rangle,|\overline{0}\rangle\}$ and perform transversal $\bar{X}$ and $\bar{Z}$ measurements. However we do not necessarily have a fault-tolerant realization of the Hadamard gate $\mathrm{H}$.

In this case the problem of constructing fault-tolerant single qubit Clifford gates can be reduced to the problem of preparing the encoded $|\overline{+i}\rangle \propto|\overline{0}\rangle+i|\overline{1}\rangle$ ancilla [31]. In particular, the gates $\mathrm{S} \propto \exp (-i \pi Z / 4)$ and $\mathrm{Q} \propto \exp (+i \pi X / 4)$ generate the single-qubit Clifford group and can be implemented given a $|+i\rangle$ ancilla, see Figure 1 and Figure 2

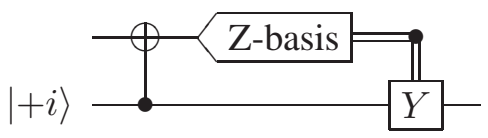

Figure 1: The S gate using a $|+i\rangle$ ancilla.

An encoded $|\overline{+i}\rangle$ ancilla can be produced using the method of injection-and-distillation [15, 14]. The distillation procedure for distilling an unencoded $|+i\rangle$ from seven unencoded $|-i\rangle$ is shown in Figure 3 In 


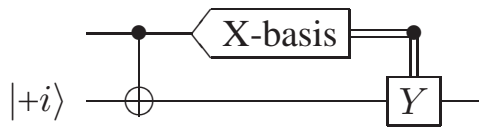

Figure 2: The Q gate using a $|+i\rangle$ ancilla.

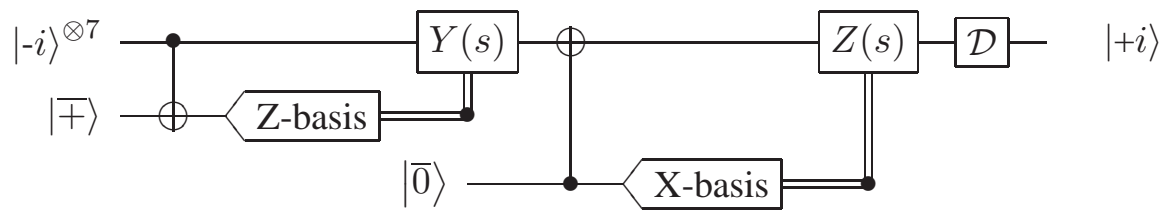

Figure 3: A circuit for the distillation of a $|+i\rangle$ ancilla from seven noisy $|-i\rangle$ ancilla's using the [[7,1,3]] Steane code. Steane error-correction (see also Figure 5) is performed on seven unencoded qubits $|-i\rangle$. Based on the $X$ and $Z$ syndromes of the Steane code the errors are corrected, except that the $X$ errors are corrected using $Y$ operators. The last step is to decode the Steane code to yield a single $|+i\rangle$ ancilla. The circuit can be found by starting from a deterministic distillation procedure for $|+\rangle$, applying an $S$ gate to the output, and conjugating it back to the input. The order of error corrections is important since the second CNOT must be replaced by a controlled-Y before the order can be reversed.

order to perform this distillation procedure in encoded form, one can generate an encoded noisy ancilla $|-\bar{i}\rangle$ using Knill's idea of injecting a state in the code. The distillation circuit is then performed in encoded form.

\section{Error Correction Circuits}

Locations in a quantum circuit are defined to be gates, single-qubit state preparations, measurement steps, or memory (wait) locations. After one level of encoding, every location (denoted as 0-Ga) is mapped onto a a rectangle or 1-rectangle (1-Rec), a space-time region in the encoded circuit, which consists of the encoded gate (1-Ga) followed by error correction (1-EC), as shown in Fig. 4. For transversal gates, the 1-Ga consists of performing the $0-\mathrm{Ga}$ 's on each qubit in the block(s).

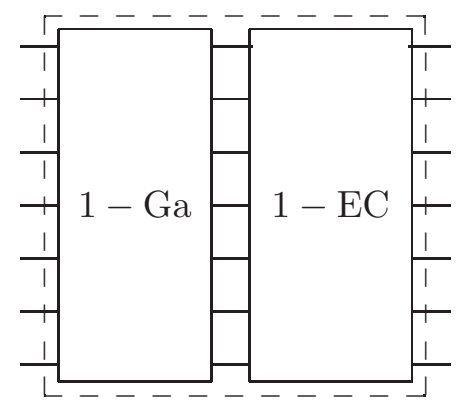

Figure 4: A 1-rectangle (1-Rec), indicated by a dashed box, which replaces a single-qubit 0-Ga location. The 1-Rec consists of the encoded fault-tolerant implementation of the $0-\mathrm{Ga}$ (1-Ga) followed by an error correction procedure (1-EC).

For the fault-tolerance analysis one also defines an extended 1-Rec or ex-Rec which consists of a rectangle along with its preceding 1-EC(s) on the input block(s). Let us now discuss the circuits for error correction.

Steane error correction for CSS codes (Steane-EC) is schematically shown in Figure 5 The $|\overline{0}\rangle$ and $|\mp\rangle$ ancilla blocks in Fig. 5 can be prepared in the following way. First $n$ qubits are encoded using circuits 


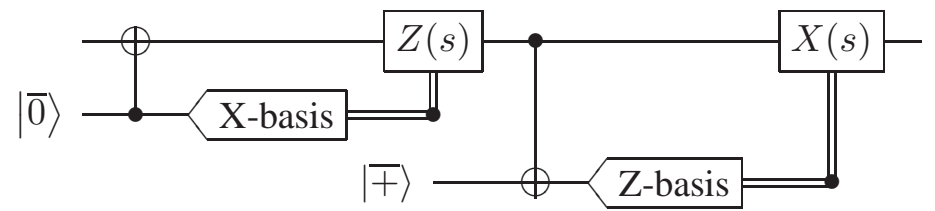

Figure 5: Steane's error correction method for CSS codes involves coupling two encoded and verified ancilla's to a block of data qubits. The ancilla qubits are then measured in the $Z$ - or $X$-basis and the syndrome $s$ is determined. From the syndrome $s$ the corresponding $Z$ or $X$ error is determined and the data qubits are corrected.

derived from the generator matrix of a classical coset code of $C_{2}^{\perp}$, see Appendix $\mathrm{A}$ for details and definitions. The memory locations in the encoder are determined using Steane's Latin rectangle method [32], discussed in more detail in Appendix A.1 Then the ancillas pass through a verification circuit. This error detection circuit measures the $X$ and $Z$ stabilizer generators of the encoded state some number $R$ of times. For a $|\overline{0}\rangle$ ancilla each round is given by the circuit in Figure 6 For dual-containing codes, the Hadamardconjugate of the circuit is used for a $|\overline{\mid}\rangle$ ancilla. If we detect any errors in any of the $R$ rounds, the encoded state is rejected. Otherwise the state is accepted and used for syndrome extraction. We will consider $L$ preparation attempts per ancilla and in our studies we will vary the parameters $R$ and $L$, giving rise to different overheads.

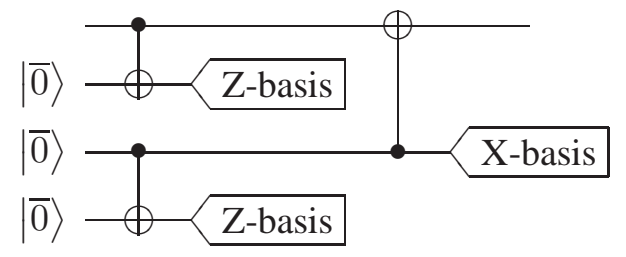

Figure 6: The ancilla verification circuit for one round of error detection. First, the top encoded ancilla is verified against $X$-errors and it is determined whether $\bar{Z}=+1$. If no $X$-errors are detected and $\bar{Z}=+1$ the encoded ancilla is then verified against $Z$-errors using an ancilla $|\overline{0}\rangle$ which is itself verified against $X$-errors.

\subsection{Specific Code Considerations}

Specific properties of a quantum code can often be used to simplify the error-correcting circuits. This section discusses each family of codes and the optimizations we have implemented or the code properties that have been used to modify the EC and 1-Ga circuits.

In general, we have opted to focus on the error-correcting properties of the codes rather than the possible simplifications to the Steane-EC network. One of the reasons for this approach is that it is not clear whether verification circuits that perform the minimal number of checks are superior to verification circuits that perform many thorough tests. Furthermore, changes to the network are difficult to parameterize and systematically study because there are many possible choices and few are clearly the best. In addition, we believe that the overall trends observed in this paper are not altered by omitting these optimizations.

Reichardt has suggested a generic optimization that uses different encoders for each logical ancilla in the verification circuit [34]. This optimization can reduce the number of necessary rounds of verification and possibly decrease the probability of correlated errors at the output of the verification circuit, conditioned on acceptance. We do not use this optimization for any of the codes in this study.

The Steane and Golay codes are constructed from perfect classical codes. Perfect codes have the property that every syndrome locates a unique error of weight $w \leq t$. As Ref. [1] observed, some parts of the 
error detection circuit can be removed for a CSS code constructed from perfect classical codes and the construction remains strictly fault-tolerant. Again we do not use this optimization.

For the Bacon-Shor codes we don't use Steane's Latin rectangle encoding method, but rather the simpler method described in [19]. We do use the standard verification method for the bigger BS codes and not the simpler verification method in [19].

For the surface codes we consider both Steane-EC and Shor-EC to understand their effects on the threshold. We use Shor-EC using bare ancillas as in [12]. This is fault-tolerant for surface codes on a $5 \times 5$ lattice or larger as long as the syndrome measurements are repeated enough times. The number of repeated measurements could in principle be varied, but we choose to repeat the measurements $\ell$ times for a $\ell \times \ell$ surface code, following [12].

The $[[49,1,9]]$ concatenated Steane code is one of the CSS codes in our study whose network deviates from the construction described in the previous section. The preparations of $|\overline{0}\rangle$ and $|\overline{+}\rangle$ do not include a verification circuit. Instead each 7-qubit block has an error detection step after each [[7,1,3]]-encoded logical gate [34]. A 49-qubit ancilla is rejected if any of these error-detections detects an error. This implies that any single fault will be detected, so the circuit is fault-tolerant. In fact, any pair of faults is also detected, so that a third order event is necessary to defeat the error-detections. This way of using $[[49,1,9]]$ is the one which Reichardt proposed. Unlike in his simulations we restrict ourselves to a finite number of ancilla preparation attempts $L$, since we care about the total overhead.

The polynomial codes that we consider are non-binary codes over $2^{m}$-dimensional qudits. We can choose the parameters of these codes so that when we consider each qudit as a block of $m$ qubits, the Fourier transform and controlled-SUM gates are implemented by bitwise application of Hadamard and CNOT, respectively. In this setting, the code is simply a binary CSS code encoding $m$ qubits which is constructed from a non-binary dual-containing classical code by concatenating using a self-dual basis of $G F\left(2^{m}\right)$. The advantage of such a construction is that we can decode the syndromes as if they were vectors over $G F\left(2^{m}\right)$, allowing us to correct more higher-order errors than we could otherwise correct as a binary code. To use this advantage, we do not need to change the way we construct the rectangles at all, only the way we interpret the classical measurement outcomes.

$[[5,1,3]]$ is the smallest distance-3 quantum error-correcting code and it is a perfect quantum code. Gottesman has shown how to compute fault-tolerantly with this code [17], and there have been some numerical studies of logical error rates using Shor-EC [16]. To our knowledge the threshold for this code has never been determined. Unfortunately, there are no two-qubit transversal gates for $[[5,1,3]]$, so it is necessary to construct a two-qubit gate from a three-qubit gate such as the $\mathrm{T}_{3}$ gate. The gate $\mathrm{T}_{3}$ is defined by the following action on Pauli operators: $X I I \rightarrow X Y Z, I X I \rightarrow Y X Z, I I X \rightarrow X X X, Z I I \rightarrow Z X Y, I Z I \rightarrow$ $X Z Y, I I Z \rightarrow Z Z Z$. This gate is a Clifford gate that can be combined with stabilizer-state preparations and transversal Pauli measurements to yield any gate in the Clifford group [17]. Specifically, CNOT, S, and Cyc gates (and their inverses) can be constructed from the $\mathrm{T}_{3}$ gate in this way. Here $\mathrm{Cyc}=\mathrm{SHSH}$ acts as $X \rightarrow Y \rightarrow Z \rightarrow X$. The fault-tolerant implementation of $\mathrm{T}_{3}$ is shown in Figure 7

The $[[5,1,3]]$ construction also differs from other CSS constructions because we use Knill (or teleported) error correction (Knill-EC) [10]. In our study we will simulate the extended $\mathrm{T}_{3}$-rectangle assuming that the logical Bell pairs of Knill-EC are perfect. We do this since it is simpler and shows that even using perfect logical Bell pairs the threshold is not very good, see Section 5] For [[5,1,3]] the $R$ and $L$ parameters are replaced by $N C$ and $N B$, denoting the number of cat state preparation attempts per Pauli measurement and the number of logical Bell state preparation attempts per error correction, respectively. A circuit to prepare and verify encoded Bell pairs for Knill error correction for [[5,1,3]] is shown in Figure 16 in Appendix B.

The construction for the $[[15,1,3]]$ Reed-Muller code is entirely standard. Since this code is not constructed from a dual-containing classical code, the $|\overline{0}\rangle$ and $|\overline{\mid}\rangle$ encoders are not simply related by a transver- 


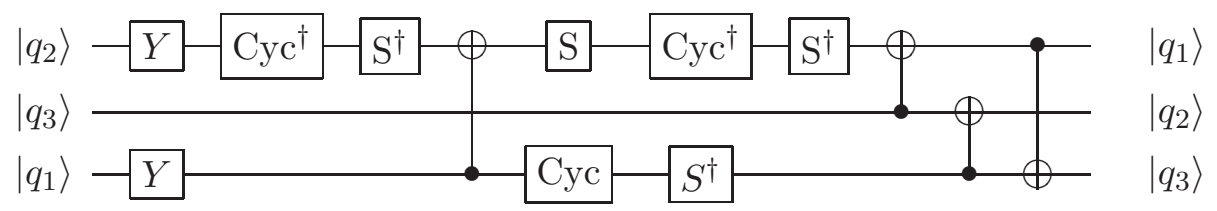

Figure 7: The encoded implementation of $\mathrm{T}_{3}$ (with an additional permutation of the blocks $q_{1}, q_{2}, q_{3}$ ) using the gates CNOT, S, Cyc and Y (and inverses). Each gate in the circuit is applied transversally. The circuit is only a logical operation after completing all of the gates, i.e. CNOT and $\mathrm{S}$ are not valid transversal gates for $[[5,1,3]]$.

sal Hadamard gate. For the same reason, the code can correct more $X$ errors than $Z$ errors. The most interesting feature of this code is that $\mathrm{T}$ is a transversal gate [23, 35], but this does not enter directly into our analysis of the threshold.

\section{The Aliferis-Gottesman-Preskill (AGP) Method}

According to [1] a rectangle is correct if the rectangle followed by an ideal decoder is equivalent to the ideal decoder followed by the ideal gate $(0-\mathrm{Ga})$ that the rectangle simulates:

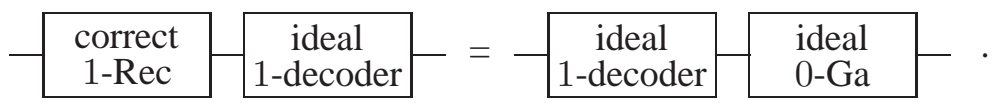

As said before, an extended rectangle (ex-Rec) consists of a 1-Ga along with its leading and trailing errorcorrections. The extended rectangles make an overlapping covering of the circuit. A set of locations inside an ex-Rec is called benign if the 1-Rec is correct for any set of faults occurring on these locations. If a set of locations is not benign, it is malignant. The design principles of strict fault-tolerance are described in pictures in Sec. 10 of [1]. If these properties hold for the 1-Ga and 1-EC, these gadgets for a [[n, 1, $d]]$ code with $t=\lfloor(d-1) / 2\rfloor$ are called $t$-strictly fault-tolerant. The important consequence of these conditions is that for a $[[n, k, d]]$ code with $t$-strictly fault-tolerant constructions one can show that any set of $t$ or fewer locations in the ex-Rec is benign. A construction is called weakly fault-tolerant when, for a code that can correct $t$ errors, sets of $s<t$ locations can be malignant. Weak fault-tolerance is a useful concept in optimizing thresholds since weakly fault-tolerant circuits can be more compact than strictly fault-tolerant circuits, hence allowing for fewer fault locations and a potentially better threshold. On the negative side, weak fault-tolerance allows some low-weight faults to be malignant but if the number of such faults is small then the threshold is not much affected.

All our fault-tolerant schemes are 1-strictly fault-tolerant implying that single faults can never be malignant. More precisely, any single fault in a 1-EC or a $1-$ Ga never propagates to become a weight- 2 error in a block. In Steane-EC when we prepare ancillas with at least two attempts $(L \geq 2)$ and one error detection stage $(R=1)$, we eliminate malignant faults of weight 1 . For $R=1$ the $\mathrm{EC}$ is not 2-strictly fault-tolerant since there may be a pair of faults, one in each of the first two encoders, generating a high weight (possibly higher than $t$ ) error that passes the error detection circuit undetected. Since the number of these events is quite rare, they will not contribute much to the failure probability. It is possible to show that $R \geq t$ and $L \geq t+1$ is necessary for $t$-strict fault-tolerance for a code that can correct $t$ errors by continuing the same argument 2 . For a specific $t$-error-correcting code the actual values required for $R$ and $L$ depend on how each encoding circuit propagates errors.

\footnotetext{
${ }^{2}$ However, the standard verification stage would need additional error detections on the bottom ancilla pair for $R=t$ and $L=t+1$ to be both necessary and sufficient for $t$-strict fault-tolerance.
} 
Let us review why the extended rectangle is the central object in a fault-tolerance analysis. An encoded circuit where the physical gates $(0-\mathrm{Ga})$ have been replaced by rectangles can also be viewed as an encoded circuit with 0 -Ga's with a different error model. This can be achieved simply by inserting perfect decoder-encoder pairs between the rectangles, see [1]. In an ex-Rec with malignant faults, the rectangle will correspond to a faulty $0-\mathrm{Ga}$, whereas for benign faults the rectangle will correspond to a perfect $0-\mathrm{Ga}$. The reason that one has to take into account an ex-Rec and not merely a Rec is that faults in the leading 1-EC can combine with faults in the 1-Rec to produce malignant faults. For example, a single error in a leading 1-EC does not cause a failure of the rectangle in which it is contained; this error however can combine with later errors to give rise to a logical error. At the same time the presence of a logical error in the leading 1-EC which maps one codeword onto another will not affect the failure rate of the rectangle that comes after it since the state which enters this rectangle is a codeword. Hence in the extended rectangle method there is no double-counting of errors. Instead, it is an efficient method to handle the effect of incoming errors and is likely to give a very tight estimate of the threshold if no other assumptions or simplifications are present.

In principle one may think that one would also need to be careful about the effect of incoming errors into the ex-Rec; perhaps an incoming error could combine with a seemingly benign fault in the ex-Rec and give rise to an incorrect rectangle. Thus perhaps one has to consider the malignancy of sets of faults given a possible worst case input to the extended rectangle.

However, one can argue for stabilizer codes and for deterministic (to be defined below) error-correction that malignancy does not depend on incoming errors to the ex-Rec. To show this, let us first review the formalism of stabilizer codes. A stabilizer code is the +1 eigenspace of an Abelian subgroup of the Pauli group $P_{n}$ which contains all $n$-qubit tensor-products of the Pauli operators $\{X, Y, Z, I\}$. The normalizer $N(S) \subseteq P_{n}$ of $S$ is defined as $N(S)=\left\{E \mid \forall s \in S, E s E^{\dagger} \in S\right\}$. For Pauli operators (which either commute or anti-commute with each other) $N(S)$ is the simply the group of Pauli operators that commute with any element in $S$. Any element of $N(S) \backslash S$ is a logical operator mapping codewords onto each other. All other Pauli operators $P \notin N(S)$ anti-commute with at least one element in $S$ and map a code word outside the code space indicated by a non-zero syndrome. Thus the Pauli group $P_{n}$ can be partitioned into cosets of $N(S)$ and each of these cosets is labeled by a different syndrome. The lowest-weight member of each coset is called the coset leader. Standard syndrome decoding finds, for each given syndrome, a coset leader with lowest weight and chooses this as the error correction. Thus the low-weight (non-degenerate) correctable errors correspond to distinct syndromes whose coset leader corrects the error. For high-weight errors $E_{i}$, all we can say is that $E_{i} E_{\text {correct }} \in N(S)$ since $E_{i}$ and $E_{\text {correct }}$ have the same syndrome.

Now let us consider the issue of incoming errors to an ex-Rec and assume the following properties of stabilizer error correction. First, we assume that the part of the 1-EC circuit which couples any ancillas to the incoming data is deterministic, i.e. does not depend on any incoming error on the data. The choice of which ancillas to couple may depend on some error detection or ancilla verification. This property holds for many but not all error-correction circuits; it does not hold, for example, when the number of repetitions of syndrome extraction depends on the value of these syndromes. This property does hold for the circuits used in this paper. Furthermore, given a stabilizer $S$ and the incoming error $E_{\text {in }}$ on an encoded state, let the 1-EC be such that the syndrome of the 1-EC uniquely determines in which coset of $N(S)$ in the Pauli group the error $E_{\text {in }}$ lies. In this sense the 1-EC must be complete error correction for the code that is used. For example, if for a CSS code the 1-EC only does $Z$ error correction whereas $X$ errors can map the state outside the code space, the syndrome information effectively partitions the Pauli group into cosets of $N\left(S_{X}\right) P_{n}(X)$. Here $P_{n}(X)$ is the subgroup of Pauli operators that only contain $X$ and $I$ and $N\left(S_{X}\right)$ is the normalizer of the stabilizer subgroup $S_{X}$ with only $X$ and $I$ Pauli operators. In this case the syndrome does not uniquely assign the incoming error to a coset of $N(S)$. Thirdly, upon any incoming error $E_{\text {in }}$ a perfect 1-EC determines a syndrome that corrects $E_{\text {in }}$ modulo a logical error (given by an element in $N(S)$ ). This is a basic property of stabilizer error correction as described above. 
Let then the incoming state to an ex-Rec be a state in the code-space of the stabilizer with an additional error $E_{\text {in. }}$. We want to show that the state that comes out of the leading 1-EC is again some state in the code space with an additional error $E_{\text {out }}$ that only depends on the errors inside the 1 -EC, $E_{\text {ec }}$, i.e $E_{\text {out }}=f\left(E_{\text {ec }}\right)$ where $f$ is independent of $E_{\text {in }}$. Any 1-EC circuit for stabilizer codes can be implemented with Clifford gates. Given an incoming error $E_{\text {in }}$ and error inside the 1-EC $E_{\text {ec }}$, it follows (because a 1-EC for any stabilizer code can be implemented with Clifford gates) that the 1-EC has syndrome $s\left(E_{\text {in }} h_{1}\left(E_{\text {ec }}\right)\right)$ where $h_{1}$ is a function independent of $E_{\text {in }}$. Based on the syndrome the correction step will be some $E_{\text {correct }}=E_{\text {in }} h_{1}\left(E_{\text {ec }}\right)$ $\bmod N(S)$. Before error correction the data has error $h_{2}\left(E_{\mathrm{ec}}\right) E_{\mathrm{in}}$ where $h_{2}\left(E_{\mathrm{ec}}\right)$ is the part of $E_{\mathrm{ec}}$ that has propagated to the data. After error correction the data thus has error $h_{2}\left(E_{\mathrm{ec}}\right) h_{1}\left(E_{\mathrm{ec}}\right) \bmod N(S)$. We strip off the logical error in $N(S)$ and identify $E_{\text {out }}=h_{2}\left(E_{\text {ec }}\right) h_{1}\left(E_{\text {ec }}\right)$. Note that when the EC is not deterministic, the functions $h_{1}$ and $h_{2}$ can depend on $E_{\text {in }}$.

We discuss the explicit decoding of the error syndromes for each code in Appendix B.

\subsection{Monte-Carlo Implementation of Method}

Given the AGP method the numerical problem to be solved is whether a Rec is correct given a set of faults in the ex-Rec containing it. This set of faults is generated using depolarizing noise with error probability $p_{0}$ for each location in the circuit. We calculate the failure rate of the ex-Rec, i.e. the probability that the Rec is not correct, for fixed $R$ and $L$. This implies that sometimes there are no verified ancillas available for a 1-EC. If this happens for any of the 1-ECs inside the extended rectangle, we call this a failure of the extended rectangle. We do this for all codes except for Reichardt's use of [[49,1,9]]. The reason for this exception is that for Reichardt's method the failure rate of ancillas may be rather high. If we let failure of having verified ancillas in the leading 1-EC determine failure of the rectangle after this 1-EC, we are possibly double-counting errors. Hence in Reichardt's method we replace any failed ancillas in the leading 1-EC by a perfect ancilla and do not call failure. As the results show, even under this assumption, the [[49,1,9]] concatenated code with error-detection and finite resources is not a great performer.

In general, our assumption on the effect of failed ancilla preparations may make our estimates for the pseudo-threshold for the EC circuits slightly more pessimistic.

We will estimate the failure rate of a CNOT ex-Rec, since this is by far the biggest circuit among the Clifford ex-Recs. As we argued in Section 2.3, the non-Clifford (and possibly other Clifford) gates will be implemented via injection-and-distillation so that their implementation will not affect the threshold. Pauli gates are not applied within a Clifford ex-Rec because they can be stored in classical memory as the Pauli frame and applied only prior to the execution of non-Clifford gates.

Given a fixed $R$ and $L$, we will estimate the failure rate $p_{1}\left(p_{0}\right)=\frac{N_{\text {fail }}}{N}$ where $N_{\text {fail }}$ is the number of Monte-Carlo samples that fail (i.e. the number of times we simulate the extended rectangle with randomly generated faults and observe that the rectangle is incorrect) and $N$ is the total number of runs. With high probability this estimated $p_{1}$ lies within one standard deviation of the real $p_{1}$. In this way we collect data points $p_{1}\left(p_{0}\right)$ for different values of $p_{0}$. We then take these points as the mean of a normal distribution for each $p_{0}$. We sample from these normal distributions and for each set of samples we determine a small degree polynomial $p_{1}\left(p_{0}\right)$ fitting the samples. The equation $p_{1}\left(p_{0}\right)=p_{0}$ gives us a sample of the threshold and we put an error bar on this result by calculating the standard deviation of the obtained threshold samples.

The way we test for correctness of a rectangle for a given pattern of faults in the ex-Rec is as follows: Let $E_{\text {out }}$ be the outgoing error of the leading 1-EC. We use syndrome decoding to determine the coset leader $E_{\text {lead }}$ corresponding to the coset of $N(S)$ in the Pauli group of this $E_{\text {out }}$. We propagate this $E_{\text {lead }}$ through the rectangle, let $f\left(E_{\text {lead }}\right)$ be the outgoing error on the data. We follow the rectangle by an ideal decoder and let $E_{\text {correct }}$ be the correction suggested by the ideal decoder. Then we test whether $E_{\text {correct }} f\left(E_{\text {lead }}\right)$ commutes with both $\bar{X}$ and $\bar{Z}$. If it does, we infer that no logical faults occurred, hence the rectangle was 
correct. Otherwise we call failure.

An alternative way of using the AGP correctness criterion is to count or sample malignant fault sets. This method is advantageous if one wants to estimate the threshold for worst-case adversarial noise. In such an application, one fixes the number of faults and counts or samples how many sets with this fixed number of faults are malignant. The failure rate $p_{1}$ is a polynomial in $p_{0}$ with factors that are determined using the malignant set counts (or estimates of these counts determined by sampling). For codes with large distance

this method becomes cumbersome, as the total number of possible fault-sets of size $t+1,\left(\begin{array}{c}A \\ t+1\end{array}\right)$ becomes large. Here $A$ is the total number of locations in the ex-Rec. This sampling method is difficult but still possible for the Golay code, but it is not possible for codes of higher distance.

The advantage of the malignant set counting or sampling method is that one gets an upper bound on $p_{1}$ for arbitrary values of $p_{0}$. This makes it possible to estimate $p_{1}$ even for very small values of $p_{0}$. We use the Monte-Carlo simulation to estimate $p_{1}$, but the number of samples required becomes quite large if one wants to estimate $p_{1}$ with good relative error for small $p_{0}$. In such cases we extrapolate the values for $p_{1}$ obtained from larger values of $p_{0}$, see Section 5.2 .

\subsection{Software and Computer Use}

On the website [36] one can find a set of software tools that have been developed for this and other future fault-tolerance projects. The quantum circuits for the CNOT ex-Recs based on CSS codes are highly structured and can be mechanically assembled in $O\left(n^{3}\right)$ time for block-size $n$ given the classical codes $C_{1}$ and $C_{2}$. We have used MAGMA [37] and/or GAP [38] (using the GUAVA package [39]) to construct quantum codes and compute their parameters. The code stabilizers are copied from the computer algebra programs into our circuit synthesis and simulation programs, where they are again verified to have the required commutation relations.

The simulation and circuit synthesis programs are implemented in C++ and use MPI [40] for communication during embarrassingly parallel tasks. The project is entirely open source and makes use of preexisting open source libraries such as a Galois field implementation [41] and a weighted matching algorithm [42]. Importantly, the same functions and procedures are used in the Monte-Carlo simulation. This gives us increased confidence in the simulation output.

The symmetries of the pair count matrix for some distance- 3 code circuits and the lack of single-location malignancies in all circuits strongly suggests that our circuit constructions are indeed fault-tolerant against single errors. Furthermore, we strictly check all input and intermediate results for consistency at runtime. The programs can be optimized and further improved, but we leave this to future work and encourage development by making the code publicly available [36].

The simulations were carried out on a relatively small allocation of Blue Gene L at the IBM T. J. Watson Research Center. Typically we used between 64 and 256 PowerPC $440700 \mathrm{MHz}$ CPUs. Each pair of CPUs had access to $512 \mathrm{Mb}$ of local memory. Using $256 \mathrm{CPUs}$ gave us roughly a factor of 50 speed-up over a typical single-processor desktop machine. The entire process of development and debugging took many months, but we estimate that all of the data could be retaken in several weeks with these computing resources.

\section{Results}

Tables 3, 4, 5, 6, and 7list the complete set of results of our studies. Our results are obtained assuming that all locations including memory locations suffer from noise at the same noise rate, unless specified otherwise. 


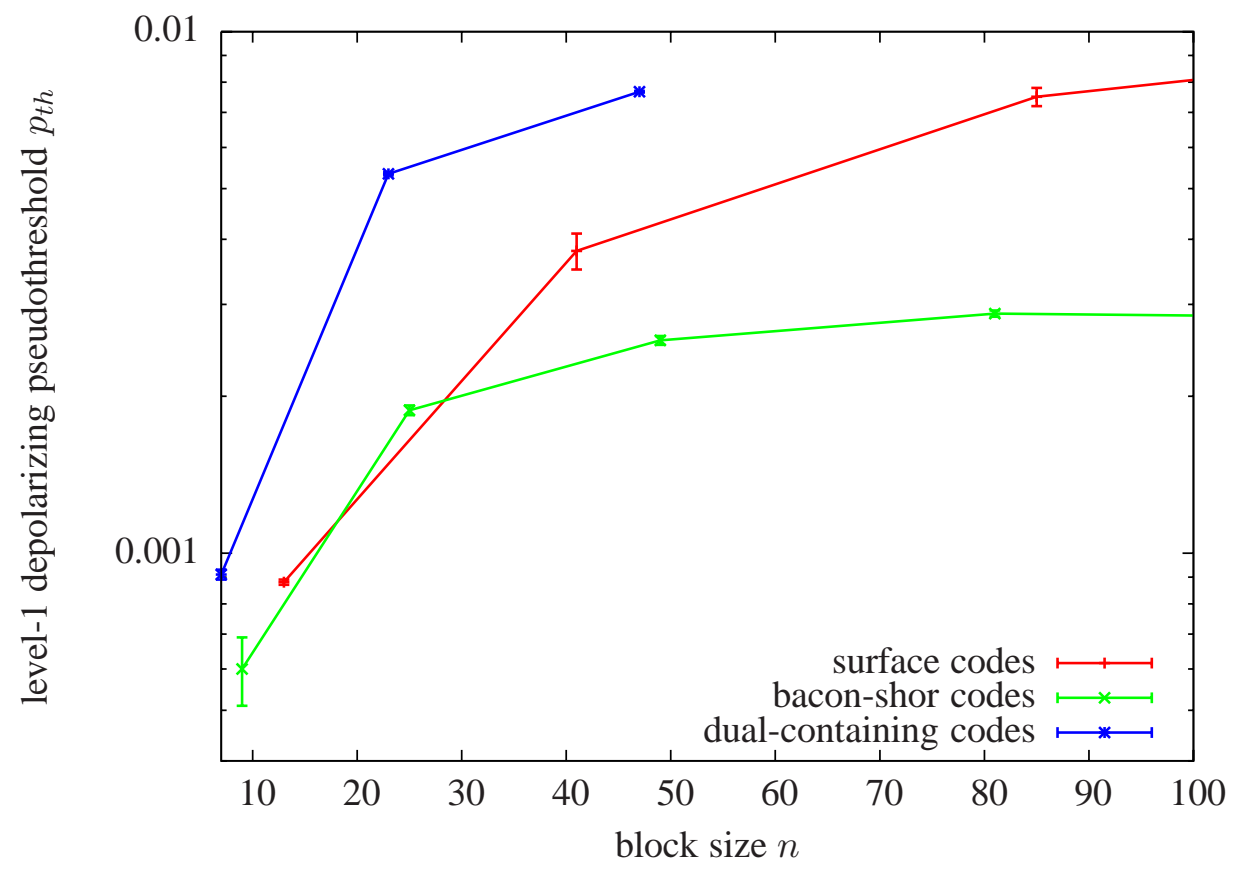

Figure 8: Level-1 depolarizing pseudo-threshold for three families of codes with perfect ancillas for SteaneEC: surface codes, dual-containing codes, and Bacon-Shor codes. This plot indicates that under no circumstances can thresholds reach $1 \%$ for the codes in our study. The data points are connected by lines merely as a guide to the eye.

\subsection{Perfect Ancillas}

In our first study, and only in this Section, we assume that ancillas for Steane error correction can be prepared flawlessly, see Figure 8 In such a scenario, the threshold is largely determined by the error-correction properties of the code (see also the analysis in [44]), in particular its (effective) distance. For families of quantum error-correcting codes in which the effective distance is linear in the block-size, we expect the threshold to be a monotonically increasing as a function of $n$, see Section 2.1 In Figure 8 and Figure 9 we have plotted the pseudo-thresholds for three families of codes: surface codes, some dual-containing codes, and Bacon-Shor codes. The surface codes and Bacon-Shor codes apparently have fairly good distance properties, even though there is some decline in the BS code family for large $n$. Figure 8 shows we cannot expect a threshold over $1 \%$ for the codes we have studied using Steane-EC - introducing noise realistically into the ancilla preparation circuits cannot increase the pseudo-threshold. Note that if we do Shor-EC on the surface codes we cannot expect thresholds exceeding about $3 \%$, see the arguments in [12].

When we assume that the logical Bell pairs of Knill's circuit can be prepared flawlessly, the level-1 pseudo-threshold of the $[[5,1,3]]$ 's $\mathrm{T}_{3}$ gate is $(2.0 \pm 0.1) \times 10^{-4} 3$. This is roughly an order of magnitude below the Steane code with perfect ancillas.

\footnotetext{
${ }^{3}$ The pseudo-threshold in this case is the point at which the failure rate $p_{1}$ of a $T_{3}$ ex-Rec is the same as the base error rate $p_{0}$ of all elementary gates in the ex-Rec.
} 


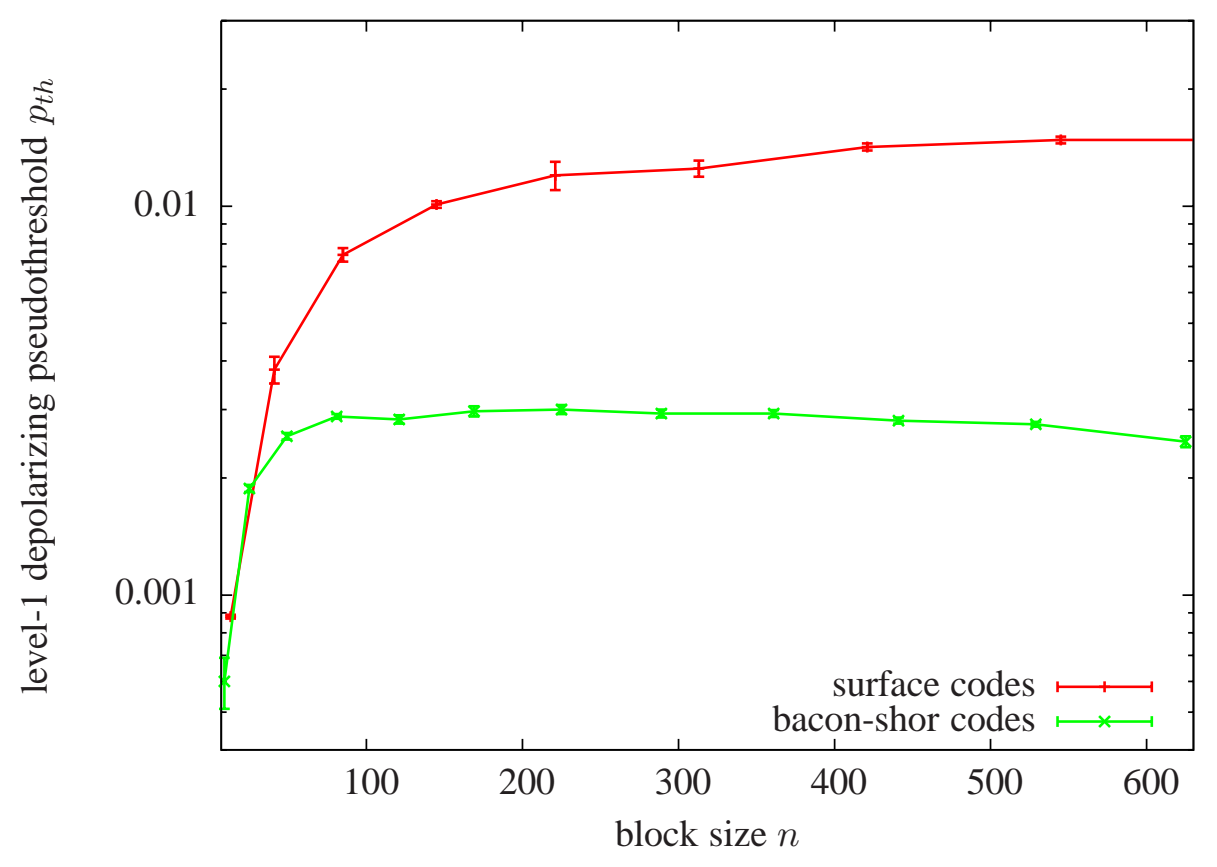

Figure 9: Level-1 depolarizing pseudo-threshold for surface codes and Bacon-Shor codes using perfect ancillas for Steane-EC.

\subsection{Pseudo-Thresholds}

In Figure 10 we tabulate for each code the maximum pseudo-threshold over the various choices of $R$ and $L$. The maximum overall pseudo-threshold $(2.25 \pm 0.03) \times 10^{-3}$ is attained by the Golay code with $L=30$ and $R=1$. The two code families, Bacon-Shor and surface, both attain a peak threshold and then decline when we use Steane-EC. The peak Bacon-Shor code is the $[[49,1,7]]$ at $(1.224 \pm 0.005) \times 10^{-3}$ with $L=9$ and $R=1$. The peak surface code (using Steane error correction) is $[[41,1,5]]$ at $(1.008 \pm 0.008) \times 10^{-3}$ at $L=30$ and $R=1$. Interestingly when we use Shor-EC for the surface codes the performance is quite different. Shor-EC does not do as well as Steane-EC for small block sizes, but for larger block size Shor-EC gives a threshold that asymptotes to a finite value, see Figure 11 For small block size the thresholds of the surface codes are not as good as of some other codes such as the Golay code and the Bacon-Shor codes.

It is clear from the data that the pseudo-threshold increases with increasing $L$. Our main interest in this study is in circuits with small overhead and hence with a relatively small number of preparation attempts $L$. In various cases the thresholds stated for finite $L$ will be thus be lower than the one in the $L \rightarrow \infty$ limit. Notably, this occurs for the $[[49,1,9]]$ code, where we expect thresholds approaching $1 \times 10^{-2}$ with many more ancilla preparation attempts [25]. In other cases one can take the perfect ancilla results in Figure 8 and the Tables as upper bounds on the $L \rightarrow \infty$ pseudo-threshold.

The use of weakly fault-tolerant circuits, i.e. small $R$ and small $L$ is meant to get improved threshold behavior for finite resources. The best performance of a code can be expected for $L \rightarrow \infty$, since one would always use ancillas which passed verification. When $L \rightarrow \infty$, it is clear that, -at least below threshold-, optimal performance is likely to be achieved when $R$ is taken as large as possible, since then an ancilla is maximally verified. However, at finite $L$, a larger $R$ will let more ancillas fail and hence increase the chance for an extended rectangle to fail (remember that we, pessimistically, call an extended rectangle failed if we don't have ancillas for EC). Hence for small $L$, small $R$, weakly fault-tolerant verification circuits can 


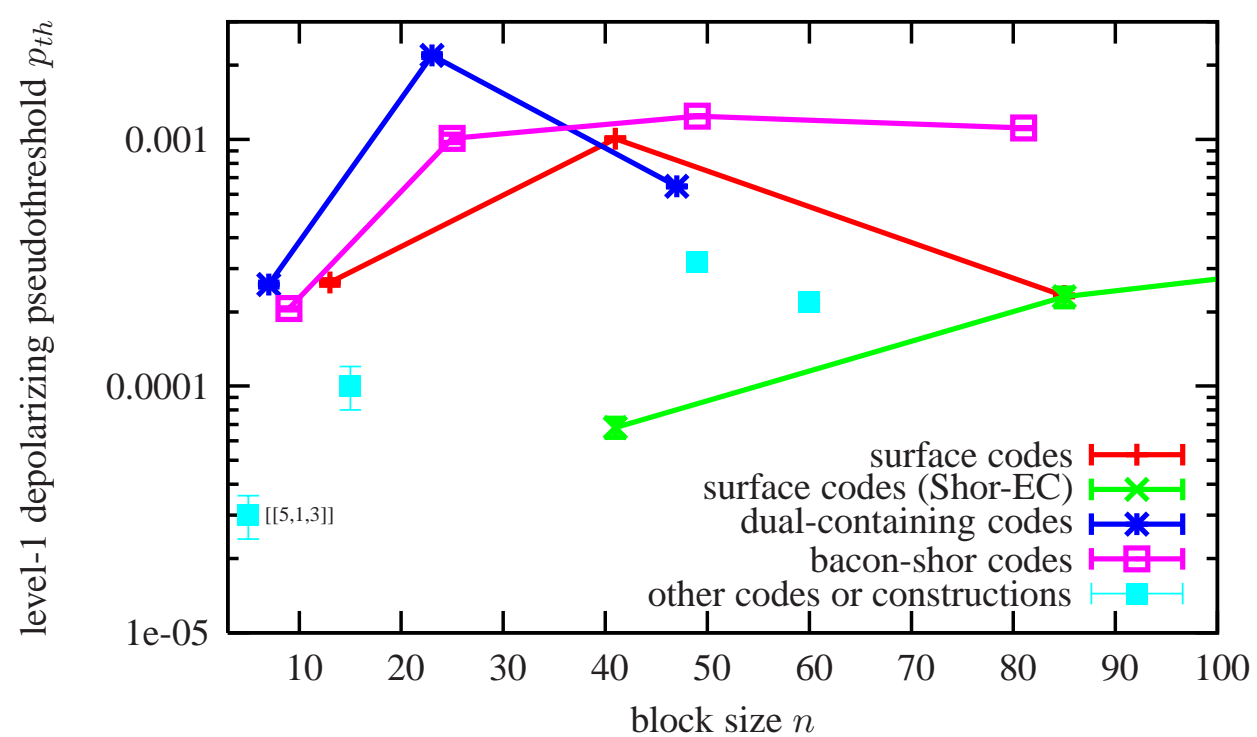

Figure 10: Level-1 depolarizing pseudo-threshold versus block size. The other codes are the $[[5,1,3]]$ non-CSS code, the $[[15,1,3]]$ Reed-Muller code, the $[[49,1,9]]$ (dual-containing) concatenated Steane code using $L=15$ attempts to prepare using error detection at level-1, and the [[60,4,10]] (dual-containing) concatenated polynomial code using $L=20$ attempts to prepare ancillas.

outperform circuits with the same $L$ and larger $R$.

\subsection{Influence of Storage Errors}

In Figure 12 we replot the pseudo-threshold versus block-size when storage error rates (on memory locations) are zero. The peak pseudo-threshold increases to $(3.33 \pm 0.02) \times 10^{-3}$. The Figure shows that storage errors do not influence the pseudo-threshold appreciably. The Bacon-Shor codes are least affected by storage errors because the encoding circuits are extremely simple. The non-CSS $[[5,1,3]]$ code is most greatly affected because storage errors can enter into the $\mathrm{T}_{3}$ gate sub-circuit, the $|\overline{0}\rangle$ encoders, and the cat-state encoders at many locations.

\subsection{Logical Error Rate versus Overhead}

The threshold is an extremely important figure of merit for fault-tolerant circuit constructions. But practically speaking, we are also interested in how quickly the error rate decreases if the initial error rates are low enough for a given overhead. Figures 13, 14, and 15 plot the probability of failure of a CNOT ex-Rec (defined in Section 4.1) versus the number of physical CNOTs in a rectangle at $p_{0}=10^{-4}$. Even though there are other measures of overhead, such as total number of qubits involved in the rectangle or the depth of the rectangle circuit, we have chosen the number of CNOTs per rectangle as an estimate for the overhead since it approximately captures the total size, i.e. depth times width, of the rectangle.

The Golay code achieves the lowest logical error rate for codes with fewer than $O\left(10^{4}\right)$ CNOT gates per rectangle, and that rate can be further reduced by increasing the number of verification rounds to $R=2$. There is a clear tradeoff between the number of physical CNOTs per rectangle and the logical error rate. We note that given the lack of code specific optimizations, the achievable overheads for various codes may 


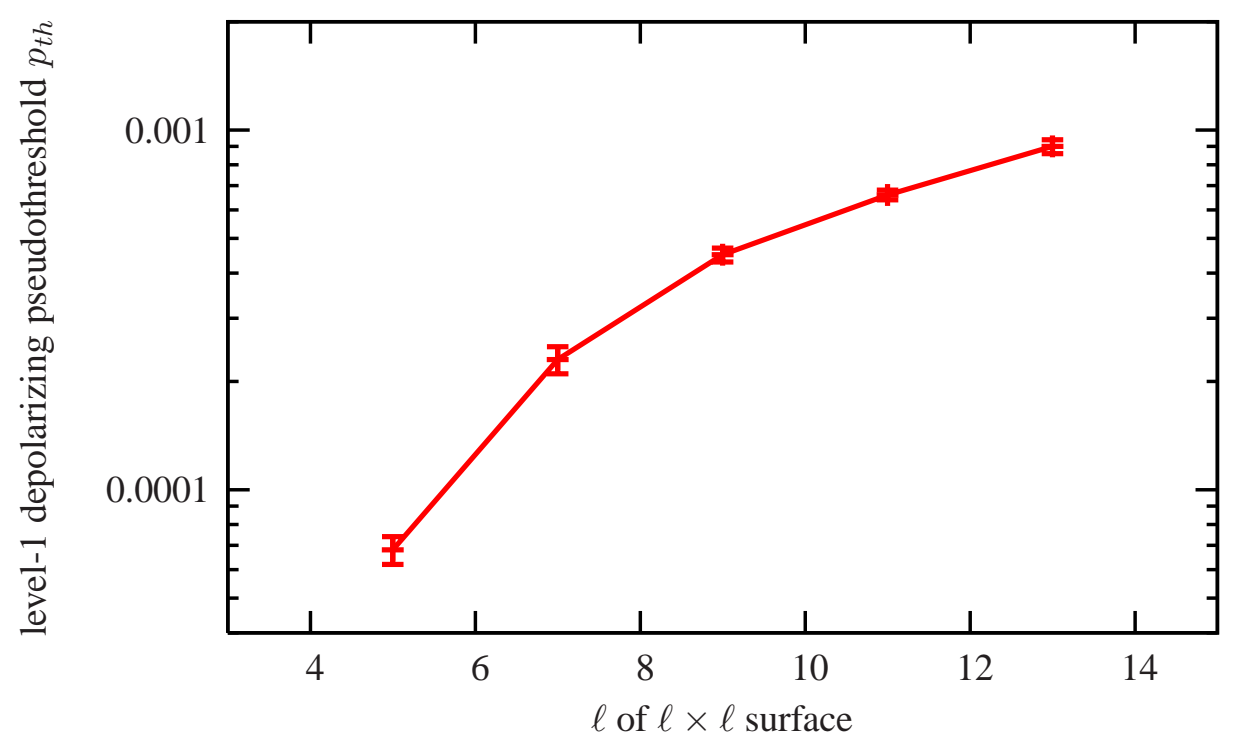

Figure 11: Surface code level-1 depolarizing pseudo-threshold versus $\ell$ for $\ell \times \ell$ surface code (the block-size $\left.n=\ell^{2}+(\ell-1)^{2}\right)$. The ex-Rec is a transversal CNOT gate with $\ell$ sequential Shor-EC steps per EC. The pseudo-threshold increases with $\ell$ and is expected to approach a constant value in the limit of large $\ell$, unlike the other codes in this study.

be somewhat less than what is estimated here. For the Golay code and the Bacon-Shor codes for example, the overhead may come down by at least a factor of 2 by using simplified verification circuits. Viewed an a log-scale such decrease in resources is relatively small. We also see in Figure 14 that the approximate expression for the failure rate, Eq. (1), gives a pretty good estimate of the actual failure rate.

Some of the error rates plotted in Figure 13 were extrapolated from error rates at higher values of $p_{0}$ and may only be rough indications of the actual error rates. For small values of $p_{0}$ the logarithm of the error rate $p_{1}\left(p_{0}\right)$ is expected to be approximately linear in $p_{0}$. We extrapolate from a least-squares fit to this line. Tables 3 , 4 and 5 indicate these extrapolated rates by enclosing them in square brackets. The extrapolations are only plotted for the $5 \times 5$ surface code and the $9 \times 9$ Bacon-Shor code and are plotted without errorbars for these two points 4 .

For the Golay code we have looked at the behavior of the threshold for $R=1,2,3$. One important empirical observation is the following. The pseudo-threshold can increase slightly while the logical error rate for $p_{0}=10^{-4}$ remains the same. This happens for the Golay code when $R=1$ and $L$ is increased from 10 to 20. Furthermore, the pseudo-threshold can decrease while the logical error rate decreases too. This also happens for the Golay code when $L=10$ and $R$ is increased from 1 to 2 . This suggests that the pseudo-threshold value is sensitive to higher order effects that quickly become negligible at lower error rates. Thus a desired logical error rate may be achievable with significantly fewer ancilla resources $L$ than are necessary to maximize the pseudo-threshold, provided the initial error rate $p_{0}$ is not too close to the pseudo-threshold.

In Figure 14 we have also added Knill's $C_{4} / C_{6}$ Fibonacci scheme [10] at 2 and 3 levels of encoding.

\footnotetext{
${ }^{4}$ Unfortunately, using the same method by which we obtain error estimates for our calculated pseudothresholds, we find error estimates that are an order of magnitude larger than these extrapolated values. In the few places where we make these extrapolations, the results should only be taken as rough indications of the actual error rates the codes can attain.
} 


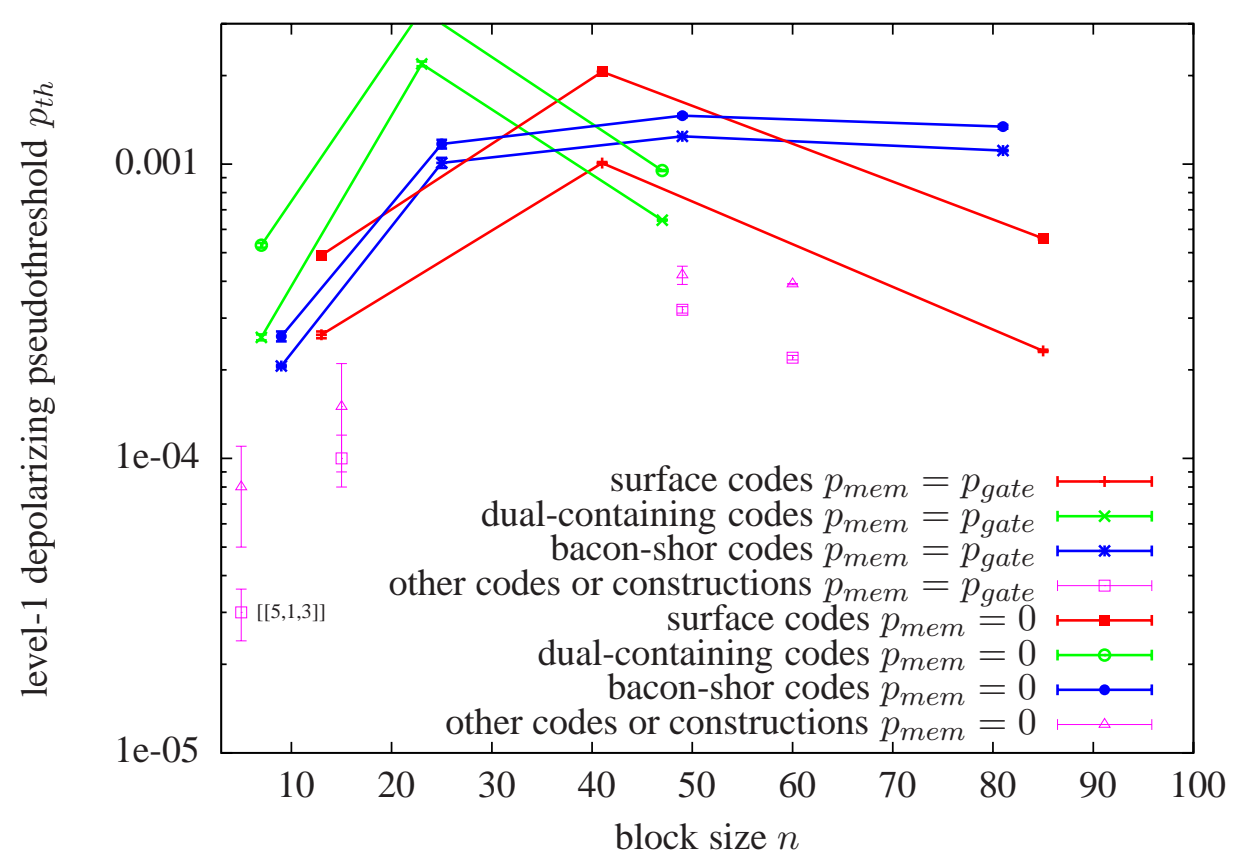

Figure 12: Pseudo-thresholds versus block size for Steane-EC and Knill-EC circuits, comparing the case where the memory failure rate equals the gate failure rate with the case where the memory failure rate is zero. Naturally the difference is smallest where we have taken advantage of simple encoders as those for the Bacon-Shor codes.

These data points are derived from his paper 5 . At level 2 the detected error rate of the logical CNOT is $(1.06 \pm 0.01) \times 10^{-5}$ and at level 3 the detected error-rate is $(2.18 \pm 0.02) \times 10^{-8}$.

The plot shows that $[[9,1,3]]$ is still better than the $C_{4} / C_{6}$ scheme in terms of overhead, but the $C_{4} / C_{6}$ Fibonacci scheme definitely beats [[7,1,3]]. The next two Bacon-Shor codes fill a void between $C_{4} / C_{6}$ level 2 and $C_{4} / C_{6}$ level 3.

For the surface codes (see Fig. 15) we note that the error rates are relatively high compared to other error-correcting codes with comparable numbers of CNOTs per rectangle. However one should remember that the circuits for the surface codes are already spatially local in two dimensions whereas the circuits for any of the other codes, for example, the Golay code, are not.

\section{Discussion}

In our study we have considered bottom codes and their performance in a bottom-top code architecture. Our best threshold around $2 \times 10^{-3}$ is seen for the Golay code, and many other codes both larger and smaller were studied and found to have much worse thresholds. An important figure of merit is the logical error rate versus overhead curve which shows that the Bacon-Shor codes are competitive with Knill's $C_{4} / C_{6}$ scheme at a base error rate of $10^{-4}$.

We have seen that the constraint of finite resources, i.e. limited $R$ and $L$ in Steane EC, can considerably and negatively impact noise thresholds. An example is Reichardt's estimate for $[[49,1,9]]$ when $L \rightarrow \infty$

\footnotetext{
${ }^{5}$ Note that his error model is slightly different from ours but we take the dominating physical CNOT error rate to be the same.
} 


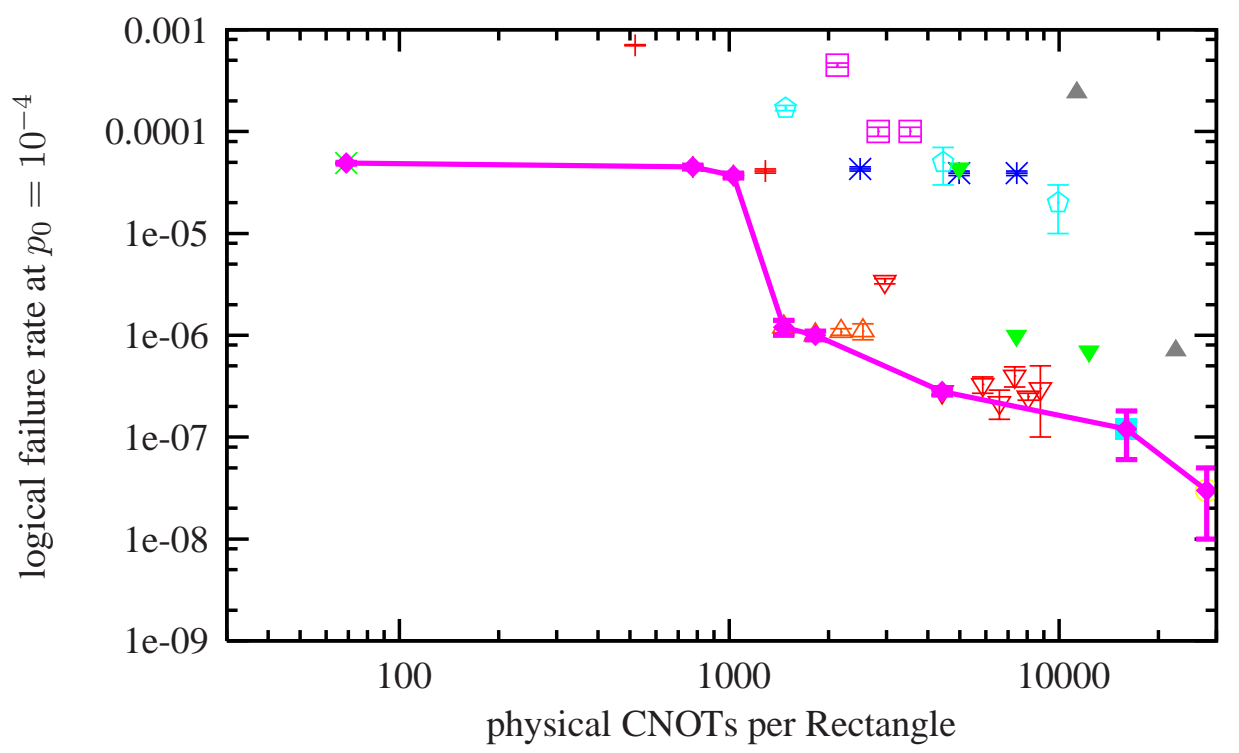

Figure 13: Level-1 logical error rate (probability of failure of a CNOT ex-Rec) versus the number of CNOTs per rectangle. The line connects the points of the best performing codes. Points with the same shape (color online) belong to the same code but have different circuit parameters. The error rates are evaluated at a fixed $p_{0}=10^{-4}$. The results C4/C6 scheme of [10] are shown for comparison.

versus our estimates for this construction at small $L$. For code families with low-weight stabilizers, Shor EC may give rise to thresholds which grow with block-size. For code families which do not have this property, e.g. general quantum $\mathrm{BCH}$ codes, the limit resource constraint on $R$ and $L$ and the complexity of the encoding circuits start pushing the thresholds down beyond some peak performance block-size.

In this landscape of codes and their performances, one of the missing players is the surface code scheme of [11] in which many qubits are encoded in one surface code and the CNOT gate is done in a topological manner. In principle, the possible advantage of this scheme is that if one uses enough space (meaning block size) one would reach the asymptotic threshold of a simple EC rectangle (no 1-Ga). We have in fact analyzed an ex-Rec where the Rec is only Shor-EC on a $\ell \times \ell$ surface and we find that this asymptotic memory threshold for $\ell \rightarrow \infty$ is about $3.5 \times 10^{-3}$. This is a factor of two lower than the number stated in [11]. For finite block size one could analyze a CNOT ex-Rec for this topological scheme just as for the other codes. Like all the other codes, the topological scheme will have a trade-off between overhead and logical error rate. It will be interesting to see whether topology and block coding provide an efficient way of using resources and how it compares to a local version of a bottom-top architecture discussed in this paper.

For a bottom-top architecture it will be important to study the performance of top codes in order to understand at what error rate one should switch from bottom to top code and what total overhead one can expect. Concerning a choice of top code we expect the following. First of all, given the constructions of [45], one can expect that a $[[n, k, d]]$ block code has a threshold comparable to a $[[n, 1, d]]$ code. Secondly, the networks in [45] show how to do logical gates on qubits inside the block codes using essentially gateteleportation and Knill-EC. One issue of concern for block codes is the complexity of the encoding circuit as a function of block size. It would be highly desirable to consider block codes with EC circuits that are linear in $n$, otherwise one would expect the threshold to decline as a function of $n$.

There is another desirable property of top codes which relates to the transversality of gates. In order to minimize overhead, it is desirable that the $\mathrm{T}$ gate is transversal for the top code. The reason is as follows. 


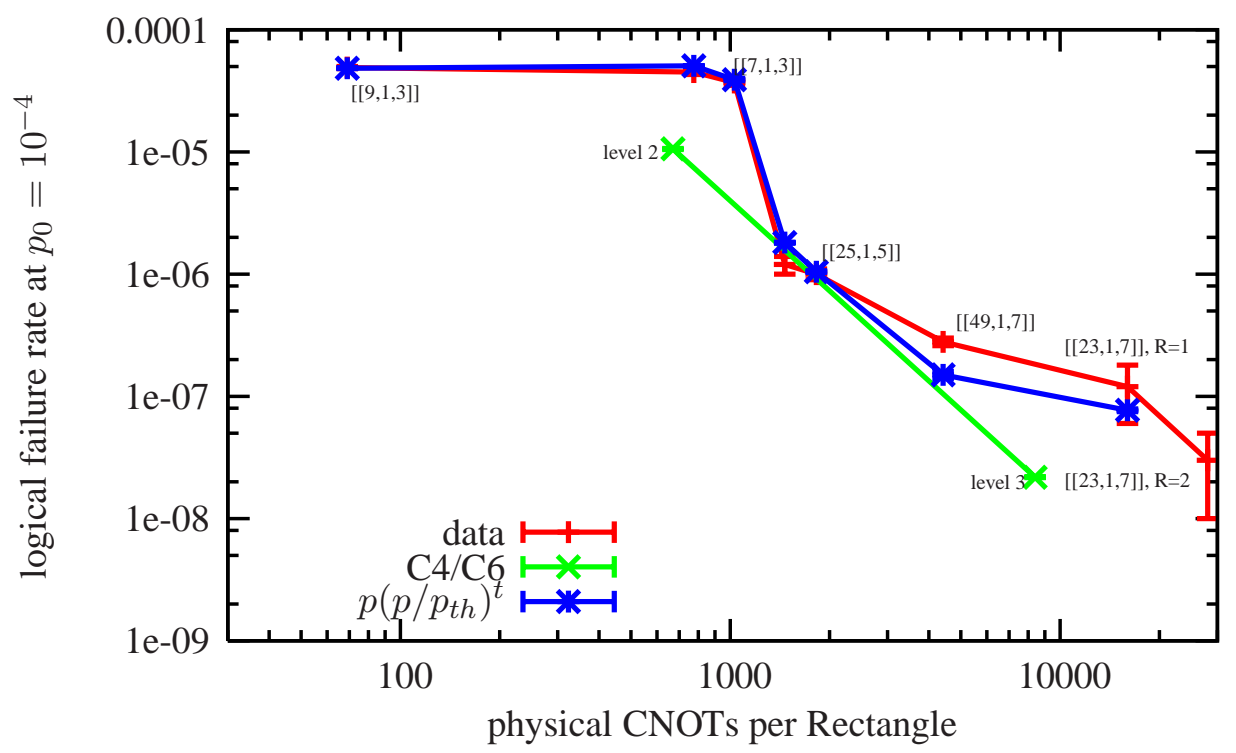

Figure 14: Level-1 logical error rate (probability of failure of CNOT ex-Rec) versus the number of CNOTs per rectangle for the best performing codes. The subset of data plotted here was chosen so that the error rate decreases monotonically with the rectangle size and there is no code with lower error rate at a given rectangle size. The error rates are evaluated at a fixed $p_{0}=10^{-4}$.

In order to have maximal freedom in picking a bottom code we will only require that it has a transversal CNOT. Thus all other gates, in particular $\mathrm{T}=e^{i \pi Z / 8}$ and the phase gate $\mathrm{S}$, should be either performed by more complicated fault-tolerant $1-\mathrm{Ga}$ or be implemented by the injection-and-distillation scheme. If the fault-tolerant circuits for these non-transversal gates have poorer thresholds than the CNOT gate, then the injection-and-distillation scheme is the preferred solution. In the injection-and-distillation scheme, the obtained error rates of the encoded and distilled ancillas will be limited by the noise rates on the Clifford gates which distill the ancillas, since the Clifford distillation circuit is not fault-tolerant. Assume we teleport the ancillas into $C_{\text {top }} \circ C_{\text {bot }}$ and get Clifford gates with $O\left(10^{-15}\right)$ error rate. Since a circuit such as BravyiKitaev distillation uses $O\left(10^{3}\right)$ gates, the error rates of the distilled ancillas can be as high as $O\left(10^{-11}\right)$. Thus by these schemes the, say, T error rate is always trailing the transversal gate error rates. But assume that the $\mathrm{T}$ gate is transversal for the top code and thus we only inject the $\mathrm{T}$ ancillas into $C_{\mathrm{bot}}$. Then even though the once encoded gate $C_{\mathrm{bot}}(\mathrm{T})$ has an error rate of, say, $O\left(10^{-4}\right)$, the twice-encoded gate $C_{\mathrm{top}} \circ C_{\mathrm{bot}}(\mathrm{T})$ will mostly likely have an error rate similar to other Clifford gates since there are very few $C_{\mathrm{bot}}(\mathrm{T})$ in the twice-encoded gate compared to the EC parts. Of course, the top code will have other non-transversal gates; for example the [[15,1,3]] code has a transversal $\mathrm{T}$ gate but not a transversal Hadamard gate. If the bottom code has a transversal Hadamard gate, we can implement a fault-tolerant $\mathrm{H}$ in $C_{\text {top }} \circ C_{\text {bot }}$ by using the fault-tolerant non-transversal gadget for the Hadamard gate in $C_{\text {top }}$ and implementing the resulting Clifford gates. This shows that there are possible constructions which would allow all gates needed for universality to be implemented with approximately the same, low, error rate, while the noise threshold of such scheme is determined by the noise threshold for the transversal Clifford gates. 


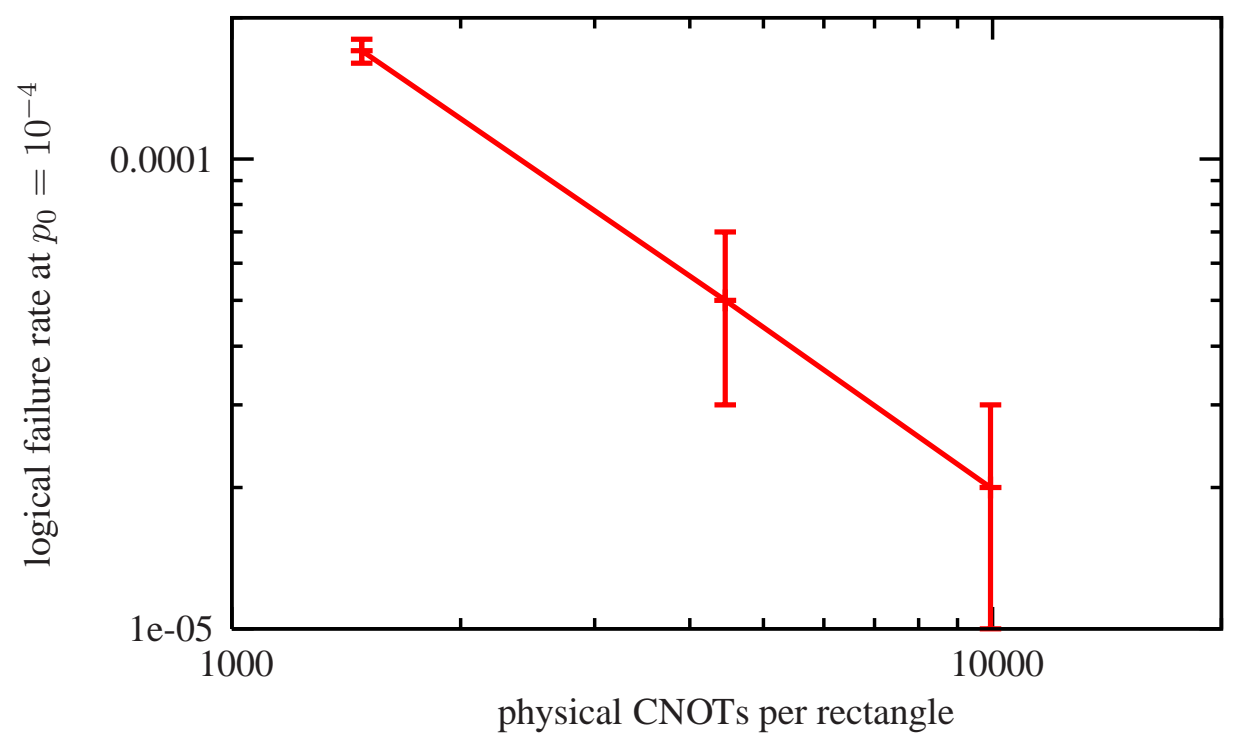

Figure 15: Level-1 logical error rate versus the number of CNOTs per rectangle for the $\ell \times \ell$ surface codes, $\ell=5,7,9$. It is expected that the error rate decreases exponentially as $\ell$ increases for fixed $p_{0}=10^{-4}$.

\section{Acknowledgements}

We acknowledge and thank Panos Aliferis, Sergey Bravyi and Austin Fowler for various fruitful discussions and insights. AC thanks Isaac Chuang for helpful discussions. DPD and BMT acknowledge support by DTO through ARO contract number W911NF-04-C-0098. AC is grateful for partial support from the Urabe Crest Project of the Japan Science and Technology Agency.

\section{A Various Aspects of Steane-EC}

A binary $[[n, k, d]]$ CSS code $\operatorname{CSS}\left(C_{1}, C_{2}\right)$ is constructed using two classical linear error correcting codes $C_{2}^{\perp} \subseteq C_{1}$ and has the codewords:

$$
|\bar{a}\rangle=\frac{1}{\sqrt{\left|C_{2}^{\perp}\right|}} \sum_{c \in C_{2}^{\perp}}|c+a\rangle \text { where } a \in C_{1} / C_{2}^{\perp} .
$$

Each row $r$ of the parity check matrix of $C_{2}$ gives the stabilizer generators $X(r)$, and each row $s$ of the parity check matrix of $C_{1}$ gives stabilizer generators $Z(s)$, where $U(r)=U_{1}^{r_{1}} \otimes \cdots \otimes U_{n}^{r_{n}}$. It is easy to check that $|\bar{a}\rangle$ is a simultaneous eigenstate of these stabilizer generators: (1) a row $r$ of the parity check matrix of $C_{2}$ must be an element of $C_{2}^{\perp}$, so adding it to each codeword in the superposition $|\bar{a}\rangle$ leaves the state unchanged, and (2) every codeword in the superposition $|\bar{a}\rangle$ is an element of $C_{1}$, so it must pass the parity checks of $C_{1}$. A basis of the $2^{k}$ cosets of $C_{2}^{\perp}$ in $C_{1}$ corresponds to logical $X$ operations $\bar{X}(a)$ on the code space because $\bar{X}(a)|\overline{0}\rangle=|\bar{a}\rangle$. Similarly, a basis of the $2^{k}$ cosets of $C_{1}^{\perp}$ in $C_{2}$ corresponds to logical $Z$ operations $\bar{Z}(b)$ since $Z(b)|\bar{a}\rangle=(-1)^{b \cdot a}|\bar{a}\rangle$. We can choose these bases such that the logical operators obey the commutation relations of the $k$-qubit Pauli group.

A special case of the CSS construction occurs when $C_{2}^{\perp}=C_{1}^{\perp}$, in which case $C_{1}$ is a dual-containing code. The $\mathrm{X}$ and $\mathrm{Z}$ stabilizer generators have identical supports and the Hadamard $\mathrm{H}$ gate is transversal. 
If in addition the weight of each stabilizer generator is a multiple of $4, C_{1}^{\perp}$ is called doubly-even and the quantum code has a transversal S gate. The code does not have any transversal gates outside of the Clifford group [46] in this case.

Steane error correction for a CSS code $C S S\left(C_{1}, C_{2}\right), C_{2}^{\perp} \subseteq C_{1}$ uses $|\overline{+}\rangle$ and $|\overline{0}\rangle$ ancilla states. These states can be encoded directly from the generator matrices of $C_{1}$ and $C_{2}^{\perp}$, respectively, according to a wellknown procedure. The generator matrix $G$ has $n$ columns and $k_{1}$ rows for $C_{1}$ or $n-k_{2}$ rows for $C_{2}^{\perp}$, and the quantum code encodes $k=k_{1}+k_{2}-n$ qubits. Gaussian elimination puts a generator matrix into standard form $G=(I \mid A)$ where $I$ is an identity matrix and $A$ is a binary matrix. The $i$ th row of the generator matrix specifies the controls and targets of $w_{i}$ CNOT gates, where $w_{i}$ is the weight of the row minus one. In the next section we discuss how to implement this circuit in a way that minimizes the number of memory locations. The depth of the resulting CNOT circuit is $w=\max \left\{w_{i}\right\}$, assuming equal cost for any pair of qubits to communicate. The number of fault locations in an encoder is summarized by the following expressions:

$$
\begin{aligned}
& A_{\mathrm{enc}}\left(n, k_{1}, k_{2}, w\right) \leq n+w \max \left(k_{1}, n-k_{2}\right), \text { no memory noise } \\
& A_{\mathrm{enc}}\left(n, k_{1}, k_{2}, w\right) \leq n+w n, \text { memory noise. }
\end{aligned}
$$

For particular states, different scaling is possible. For example, for the Bacon-Shor codes one can make the encoded ancillas using $O(n)$ 2-qubit gates. In general, any unitary stabilizer circuit has an equivalent circuit with $O\left(n^{2}\right)$ gates and $O(\log n)$ depth [48].

One method of verifying the encoded ancilla against low-weight correlated errors is to use transversal gates to perform error detection. One possible error detection method consumes three additional ancilla and uses 3 transversal CNOT gates and 3 transversal measurements. The cost of verifying is:

$$
\begin{aligned}
& A_{\text {ver }}\left(n, k_{1}, k_{2}, w, R\right) \leq R\left(3 A_{\text {enc }}\left(n, k_{1}, k_{2}, w\right)+6 n\right), \text { no memory noise } \\
& A_{\text {ver }}\left(n, k_{1}, k_{2}, w, R\right) \leq R\left(3 A_{\text {enc }}\left(n, k_{1}, k_{2}, w\right)+6 n\right)+n, \text { memory noise. }
\end{aligned}
$$

Again, these expressions assume equal cost for any pair of qubits to communicate.

Finally, we can write expressions for the total number of fault locations in a CNOT extended rectangle using Steane error correction:

$$
A(n, k, w, R) \leq 8 A_{\mathrm{enc}}\left(n, k_{1}, k_{2}, w\right)+8 A_{\mathrm{ver}}\left(n, k_{1}, k_{2}, w, R\right)+17 n .
$$

If we set $R=t$ then the total number of fault locations is $A\left(n, k_{1}, k_{2}, w, R\right)=O(w n t)$ using this method of error correction and assuming equal communication costs between qubits. In the worst case this can be $O\left(n^{3}\right)$.

\section{A.1 Latin Rectangle Method for Optimizing Encoding Circuits}

There is a simple method for minimizing the number of memory locations in an ancilla encoding circuit due to Steane. Steane puts the generator matrix $G$ of a linear binary code into standard form $(I \mid A)$ using Gaussian elimination. An encoding circuit for the logical zero state can be constructing by looking at the $A$ matrix for the code $C_{2}^{\perp}$. Every 1 in the $A$ matrix gives a CNOT gate in the encoder. The control qubits are the $1 \mathrm{~s}$ in the $I$ part of $G$ and the target qubits are the 1s in the $A$ part of $G$.

For example, we have $G=(1010101,0110011,0001111)$ for the $[7,3,4]$ code, which is the $C_{2}^{\perp}$ for Steane's [[7,1,3]] code. Transposing columns 3 and 4 gives the standard form and an $A=(1101,1011,0111)$. This means there are 9 CNOT gates in the logical zero encoder. We can assign each CNOT a time-step so that no qubit is involved in two gates at once. That constraint makes a time-step assignment the same as 
finding a partial Latin rectangle. The Latin rectangle to complete is $\left[\begin{array}{llll}? & ? & & ? \\ ? & & ? & ? \\ & ? & ? & ?\end{array}\right]$ and one possible completion is $\left[\begin{array}{cccc}1 & 2 & & 3 \\ 2 & & 3 & 1 \\ & 3 & 1 & 2\end{array}\right]$. The circuit corresponding to the time-step assignment is:

\# time 1

cnot 1,4

cnot 2,7

cnot 3,6

\# time 2

cnot 1,5

cnot 2,4

cnot 3,7

\# time 3

cnot 1,7

cnot 2,6

cnot 3,5

We have to undo the qubit permutation that occurred in the Gauss elimination to standard form, so at the end we should switch back qubits 3 and 4 . This is the smallest depth (3) that a circuit for $A$ can have. The smallest depth is the maximum row or column sum $w$ of $A$.

The problem of completing the Latin rectangle and therefore of computing the optimal time-step assignment for a matrix $A$ is equivalent to edge coloring a bipartite graph with the minimum number of colors. We construct the graph in the following way. The left set of vertices corresponds to the control qubits. The right set of vertices corresponds to the target qubits. A control and target vertex are connected by an edge if there is a CNOT between those two qubits. Assign a color to an edge to indicate what time-step we plan to do that CNOT gate. Since we cannot have two CNOT gates occur at the same time using the same qubit, all of the edges incident to a given vertex must have different colors. This means that a valid schedule corresponds to an edge coloring of this bipartite graph (bipartite because we have a set of control vertices that are only connected to target vertices, and a set of target vertices that are only connected to control vertices). By Hall's theorem [32], there is a coloring using $w$ colors, and $w$ colors is the minimum number of colors we can use. Here $w$ is maximum weight of the rows of $A$ minus 1. See [47] for an algorithm that finds an edge coloring with $w$ colors in time $O\left(n N_{\mathrm{CNOT}}\right)$. Here $n$ is the number of qubits that are to be encoded (i.e. number of vertices) and $N_{\mathrm{CNOT}}$ is the number of CNOT gates in the encoder (i.e. number of edges).

We have tested several of the encoders produced by our Latin rectangle software tool with and without memory locations by simulating them using $\mathrm{CHF}_{6}^{6}$ [48].

\section{B Syndrome Decoding}

General algorithms for constructing the classical circuits to decode measurement outcomes obtained in Steane error correction require exponential time and/or space. Therefore, we consider each code's syndrome decoder separately, essentially devising a special-purpose algorithm for each to make the decoding feasible.

Table 2 lists all of the codes we consider in this study and their syndrome decoders. There are six distinct decoding algorithms that we use to compute the error locations and type of error from the syndrome

\footnotetext{
${ }^{6} \mathrm{CHP}$ stands for CNOT, Hadamard, Phase.
} 


\begin{tabular}{l|l|} 
CODE & DECODER \\
\hline$[[5,1,3]]$ & Table Lookup \\
{$[[7,1,3]]$} & Table Lookup (cyclic) \\
{$[[9,1,3]],[[25,1,5]],[[49,1,7]],[[81,1,9]]$} & Majority \\
{$[[15,1,3]]$} & Table Lookup \\
{$[[13,1,3]],[[41,1,5]],[[85,1,7]]$} & Min. Wt. Matching \\
{$[[21,3,5]]$} & Table Lookup (cyclic) \\
{$[[23,1,7]]$} & Table Lookup (cyclic) \\
{$[[47,1,11]]$} & Algebraic [49] \\
{$[[49,1,9]]$} & Table Lookup with Message Passing \\
{$[[60,4,10]]$} & Table Lookup (cyclic)
\end{tabular}

Table 2: The decoders that we use for the codes in our study.

measurements: a generic table lookup algorithm, a table lookup algorithm for cyclic codes over arbitrary fields, a majority voting algorithm for Bacon-Shor codes, a minimum weight matching algorithm for surface codes, a simple message passing algorithm for the concatenated Hamming code, and an algebraic decoder for the $[[47,1,11]]$ quadratic residue code.

Rather than use a general table-lookup algorithm, we use a so-called Meggitt decoder which uses the fact that the polynomial codes and the Hamming, Golay, and quadratic residue (QR) codes are constructed from cyclic classical codes. Cyclic codes have a compact description in terms of a generating polynomial whose coefficients give one of the code words and whose cyclic shifts generate a basis for the code. The Meggitt decoding algorithm stores a table of syndromes and their associated error corrections [50]. For nonbinary codes such as the polynomial codes, the table stores both error locations and error-type (the so-called amplitude). Only $\left(\begin{array}{c}n \\ w-1\end{array}\right)$ syndromes need to be stored for a weight $w$ error, since one of the coordinates can be fixed by the cyclic symmetry. Finding the appropriate recovery requires at most $n$ table lookups. If we fail to find a recovery in the table, a subroutine is triggered that applies some syndrome-dependent correction mapping the state back into the code space.

For cyclic codes with larger distance where table lookup is impractical, for example [[47,1,11]], algebraic decoding techniques can be used. The generator polynomial's roots are used to compute a sequence of syndromes from which we can locate errors. $\mathrm{BCH}$ codes are easy to decode because their generator polynomials have a contiguous sequence of roots so the Berlekamp-Massey algorithm can find the error-locator polynomial whose roots give the error locations. Sometimes decoding up to the full minimum distance of the code is challenging because the generator polynomial may not have a long sequence of roots, so some syndromes are missing and the Berlekamp-Massey algorithm cannot be directly applied. In this case, unknown syndromes can sometimes be computed from algebraic equations involving the known syndromes. Algebraic decoding of the $[[47,1,11]]$ proceeds this way. For each error weight from zero to $t$, we compute any missing syndromes, construct a polynomial whose roots are the error locations, and find the roots of the polynomial. If the polynomial has enough roots, we correct those errors and stop. If we do not find enough roots for each of the locators, we return a "failed" result, triggering a subroutine that applies some syndrome-dependent correction that maps the state to some (possibly logically incorrect) state in the code space. The implementation details can be found in [49].

The Bacon-Shor codes are essentially concatenated quantum repetition codes. Since the code stabilizer is preserved by bitwise Hadamard composed with a 90 degree rotation of the square lattice, one syndrome decoder is sufficient for both $X$ and $Z$ error correction. Imagine a vector of $n^{2}$ syndrome bits placed on an $n$ by $n$ square lattice. Let $s_{x}$ be the syndrome vector for $X$ errors and $s_{z}$ be the syndrome vector for 
$Z$ errors. Let $R$ be the map on vectors of length $n^{2}$ that rotates them by 90 degrees on the square lattice. The same syndrome decoder is applied to $s_{x}$ and $R s_{z}$. The syndrome decoder decodes a variation on the classical repetition code on $n$ bits. First, the decoder computes the parity of each column of the lattice and stores each column parity as an element of a vector $p$. Next, the decoder computes the repetition code parity check $h=H p$. This parity check $H$ is expressed in standard form $\left[I_{n-1} 1\right]$ where 1 is the all ones column vector. Finally, the decoder infers the error locations from the parity check. If the weight of the parity check is greater than $t$, we must assume that the rightmost bit of $p$ was incorrect so that $h \oplus 1$ gives the error locations on the first $n-1$ bits of $p$. Otherwise, we infer that the rightmost bit of $p$ was correct so that $h$ gives the error locations on the first $n-1$ bits of $p$.

The surface code is decoded using Edmond's minimum-weight matching algorithm. The approach differs slightly depending on whether Steane-EC or Shor-EC is used but is essentially the same as [12]. Steane-EC gives a 2D matching problem whereas Shor-EC gives a 3D matching problem. The mapping from syndrome information to a matching problem is as follows.

Nonzero syndrome bits are called defects and are located somewhere in the $\ell \times \ell$ plane. We construct a complete weighted graph whose vertices represent defects and whose edge weights indicate the distance between defects. The surface code's syndrome may be such that there are lone defects which are not caused by error patterns connecting two defects, but by an error pattern connecting an edge-defect on the boundary to an inner defect. $X$ and $Z$ errors constitute separate matching problems and $X$-defects can be matched with, say, the horizontal boundaries and $Z$-defects with the vertical boundaries.

We can design an algorithm for decoding the surface code for, say, $Z$ errors, as follows:

- Imagine cutting the lattice vertically in two halves, left (L) and right (R). Let $N_{L / R}(i)$ be the number of defects in row $i$ of the left/right part of the lattice. For each row of the lattice, add $N_{L / R}(i)$ edge defects on the $i$ th row on the left (right) boundary.

- Assign the weight of the edges between any edge defects as zero and assign the distance as the weight between edge defects and inner defects.

- Compute the minimum-weight perfect matching of the graph of defects.

- The recovery operation consists of applying phase flips on the qubits that are along the edges of each pair of matched vertices in the graph.

Note that the algorithm enforces the property that the graph has an even number of vertices, so that every vertex can be matched.

The concatenated [[7,1,3]] code, that is, the [[49,1,9]] code, can be decoded to distance 7 if we treat it as a concatenated code. However, decoding the code to distance 9 requires a slight modification of the algorithm so that a simple message is passed from level-1 to level-2.

Suppose the 49 transversal measurement outcomes are organized into 7 registers of 7 bits each. We use these registers as temporary storage to compute the appropriate correction. First, we compute the level-1 syndromes for each register as we would normally do. These syndromes indicate errors $e_{i}$ in the $i$ th level- 1 register. We correct each level-1 register according to the $e_{i}$ s and "flag" those registers for which $e_{i} \neq 0$. Next, we compute the level-2 (logical) syndrome of the resulting 49 bit register, which now has trivial level1 syndrome in each 7 bit register. This level-2 syndrome indicates a logical correction $\bar{e}$ that is constant on each level-1 register (but two level-1 registers can take different values). The correction $c_{1}:=\left(\bigoplus_{i} e_{i}\right) \oplus \bar{e}$ corrects all errors of weight 4 or less, except for one problem case. This case occurs when a pair of errors occurs in one level-1 register and another pair of errors occurs in a different level-1 register. The problem is overcome by comparing the register positions where $\bar{e}$ is 1 with the positions of the flags whenever two flags 


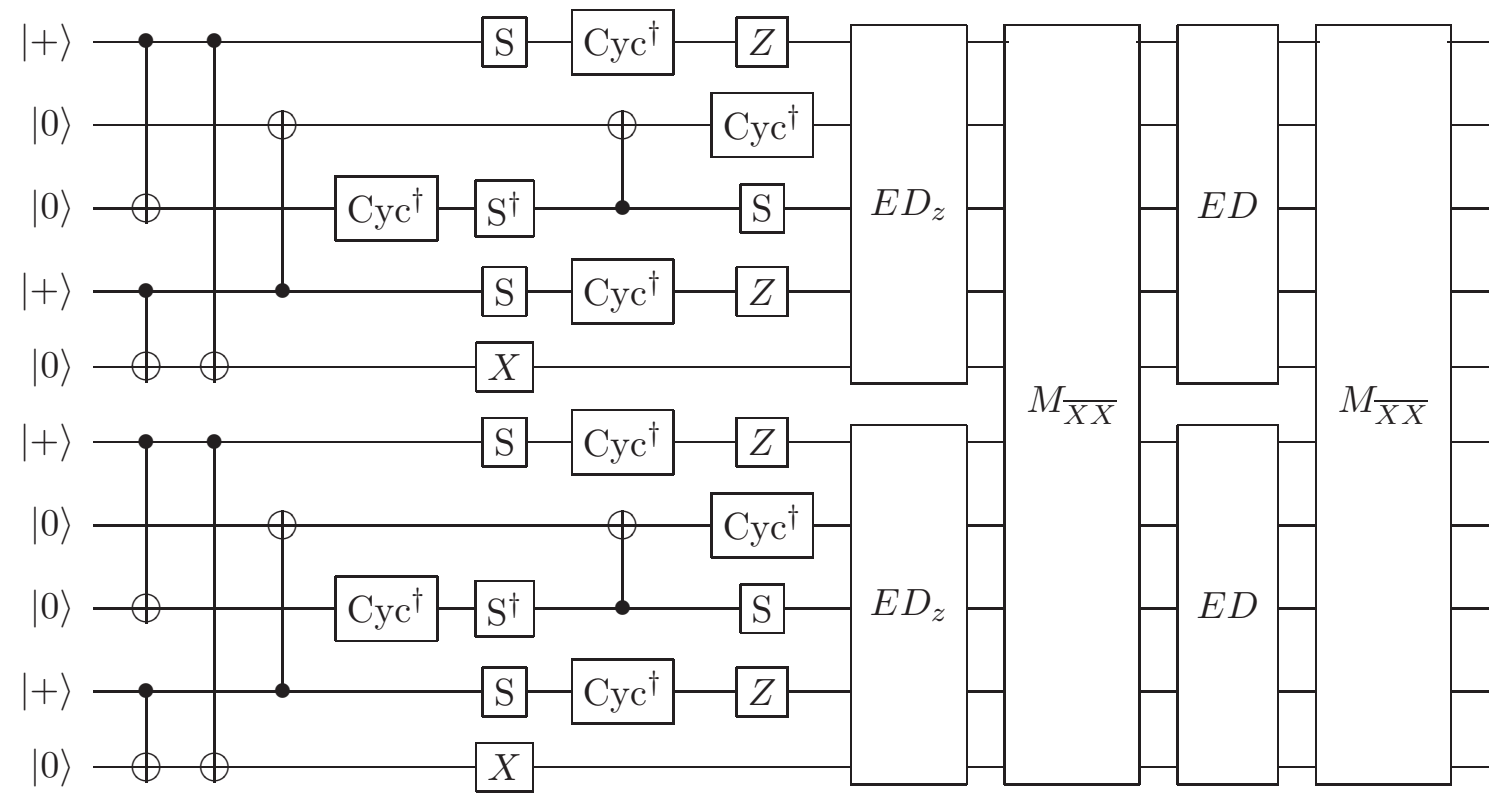

Figure 16: A fault-tolerant circuit for preparing logical Bell pairs for Knill error correction of $[[5,1,3]]$. The sub-circuit $E D_{z}$ measures the stabilizer of $|\overline{0}\rangle,\langle X Z Z X I, I X Z Z X, X I X Z Z, Z X I X Z, Z Z Z Z Z\rangle$, using 4 and 5 qubit cat states, and the sub-circuit $E D$ makes the same measurement without measuring $\bar{Z}=Z Z Z Z Z$. If any measurement outcome is nonzero, the Bell state is rejected. The sub-circuit $M_{\overline{X X}}$ measures $\overline{X X}=X^{\otimes 10}$ using a 10 qubit cat state. The Bell state is rejected if the $\overline{X X}$ measurements disagree, but if they are both 1 then $\bar{Z}_{1}$ is applied to the output Bell pair. The cat states are verified so that if a cat state is accepted then a single fault in its preparation cannot produce a correlated error.

are raised. If they disagree, apply the correction $c_{2}:=\left(\bigoplus_{i} e_{i}\right) \oplus \bar{f}$ where $\bar{f}$ is a logical correction on the flagged registers. Otherwise, apply the original correction $c_{1}$. This procedure corrects all errors of weight 4 or less and returns the input to the codespace in all cases.

The classical decoding algorithms have been tested exhaustively for all of the codes in the paper except for the large Bacon-Shor and surface codes. The algorithms were found to correct all errors of weight $t$ or less. In the case of the polynomial codes, $t$ is the number of errors the underlying nonbinary code can correct. The same decoding algorithms that were tested exhaustively for small Bacon-Shor and surface codes were used for the larger codes in those families.

\section{Data Tables}




\begin{tabular}{|c|c|c|c|c|c|c|c|}
\hline$[[n, k, d]]$ & $\mathrm{L}^{a}$ & $\mathrm{R}^{b}$ & $\mathrm{CX} / \mathrm{REC}^{c}$ & $p_{1}\left(p_{\mathrm{mem}}=0, p_{0}=10^{-4}\right)$ & $p_{1}\left(p_{\mathrm{mem}}=p_{0}=10^{-4}\right)$ & $p_{\text {th }}\left(p_{\text {mem }}=0\right)$ & $p_{\mathrm{th}}\left(p_{\mathrm{mem}}=p_{0}\right)$ \\
\hline$\overline{[[5,1,3]]}$ & 2 & 2 & 2,160 & - & - & $(3.9 \pm 0.7) \times 10^{-5}$ & $(2.5 \pm 0.4) \times 10^{-5}$ \\
\hline$[[5,1,3]]$ & 3 & 3 & 5,117 & - & - & $(9.2 \pm 0.5) \times 10^{-5}$ & $(3.7 \pm 0.3) \times 10^{-5}$ \\
\hline$[[5,1,3]]$ & 5 & 5 & 14,775 & - & - & $(9.2 \pm 0.5) \times 10^{-5}$ & $(3.3 \pm 0.6) \times 10^{-5}$ \\
\hline$[[5,1,3]]$ & 10 & 3 & 18,536 & - & - & $(8.8 \pm 0.5) \times 10^{-5}$ & $(4.3 \pm 0.3) \times 10^{-5}$ \\
\hline$[[5,1,3]]$ & 10 & 10 & 60,760 & - & - & $(8 \pm 3) \times 10^{-5}$ & $(3.0 \pm 0.6) \times 10^{-5}$ \\
\hline$[[7,1,3]]$ & 2 & 1 & 519 & $(5.34 \pm 0.07) \times 10^{-4}$ & $(7.05 \pm 0.08) \times 10^{-4}$ & $(1.85 \pm 0.05) \times 10^{-5}$ & $(1.46 \pm 0.05) \times 10^{-5}$ \\
\hline$[[7,1,3]]$ & 3 & 1 & 775 & $(2.3 \pm 0.2) \times 10^{-5}$ & $(4.5 \pm 0.2) \times 10^{-5}$ & $(3.11 \pm 0.02) \times 10^{-4}$ & $(1.98 \pm 0.01) \times 10^{-4}$ \\
\hline$[[7,1,3]]$ & 4 & 1 & 1,031 & $(1.9 \pm 0.1) \times 10^{-5}$ & $(3.7 \pm 0.2) \times 10^{-5}$ & $(4.97 \pm 0.07) \times 10^{-4}$ & $(2.56 \pm 0.06) \times 10^{-4}$ \\
\hline$[[7,1,3]]$ & 5 & 1 & 1,287 & $(1.8 \pm 0.1) \times 10^{-5}$ & $(4.1 \pm 0.2) \times 10^{-5}$ & $(5.3 \pm 0.1) \times 10^{-4}$ & $(2.58 \pm 0.06) \times 10^{-4}$ \\
\hline$[[9,1,3]]$ & 1 & 1 & 69 & - & $(4.90 \pm 0.09) \times 10^{-5}$ & $(2.6 \pm 0.1) \times 10^{-4}$ & $(2.06 \pm 0.02) \times 10^{-4}$ \\
\hline$[[13,1,3]]$ & 3 & 1 & 1,501 & - & - & $(1.59 \pm 0.04) \times 10^{-4}$ & $(0.69 \pm 0.03) \times 10^{-4}$ \\
\hline$[[13,1,3]]$ & 4 & 1 & 1,997 & - & - & $(3.81 \pm 0.07) \times 10^{-4}$ & $(1.95 \pm 0.04) \times 10^{-4}$ \\
\hline$[[13,1,3]]$ & 5 & 1 & 2,493 & - & $(4.3 \pm 0.2) \times 10^{-5}$ & $(4.9 \pm 0.2) \times 10^{-4}$ & $(2.30 \pm 0.08) \times 10^{-4}$ \\
\hline$[[13,1,3]]$ & 10 & 1 & 4,973 & $(1.8 \pm 0.1) \times 10^{-5}$ & $(3.9 \pm 0.2) \times 10^{-5}$ & $(5.1 \pm 0.2) \times 10^{-4}$ & $(2.54 \pm 0.07) \times 10^{-4}$ \\
\hline$[[13,1,3]]$ & 15 & 1 & 7,453 & $(1.9 \pm 0.1) \times 10^{-5}$ & $(3.9 \pm 0.2) \times 10^{-5}$ & $(4.9 \pm 0.1) \times 10^{-4}$ & $(2.63 \pm 0.07) \times 10^{-4}$ \\
\hline$[[15,1,3]]$ & 3 & 1 & 2,127 & $(1.3 \pm 0.2) \times 10^{-4}$ & $(4.5 \pm 0.2) \times 10^{-4}$ & $(0.86 \pm 0.03) \times 10^{-4}$ & $(0.33 \pm 0.05) \times 10^{-4}$ \\
\hline$[[15,1,3]]$ & 4 & 1 & 2,831 & $(4.9 \pm 0.7) \times 10^{-5}$ & $(1.0 \pm 0.1) \times 10^{-4}$ & $(1.5 \pm 0.6) \times 10^{-4}$ & $(1.0 \pm 0.2) \times 10^{-4}$ \\
\hline$[[15,1,3]]$ & 5 & 1 & 3,535 & $(5.8 \pm 0.8) \times 10^{-5}$ & $(1.0 \pm 0.1) \times 10^{-4}$ & $(1.8 \pm 0.2) \times 10^{-4}$ & $(1.0 \pm 0.2) \times 10^{-4}$ \\
\hline$[[23,1,7]]$ & 10 & 1 & 16,023 & $(1.1 \pm 0.3) \times 10^{-7}$ & $(1.2 \pm 0.6) \times 10^{-7}$ & $(1.14 \pm 0.05) \times 10^{-3}$ & $(1.09 \pm 0.01) \times 10^{-3}$ \\
\hline$[[23,1,7]]$ & 20 & 1 & 32,023 & $(1.2 \pm 0.4) \times 10^{-7}$ & $(9 \pm 4) \times 10^{-8}$ & $(2.33 \pm 0.02) \times 10^{-3}$ & $(1.97 \pm 0.02) \times 10^{-3}$ \\
\hline$[[23,1,7]]$ & 30 & 1 & 48,023 & - & - & $(2.98 \pm 0.04) \times 10^{-3}$ & $(2.25 \pm 0.03) \times 10^{-3}$ \\
\hline$[[23,1,7]]$ & 40 & 1 & 64,023 & - & - & $(3.33 \pm 0.02) \times 10^{-3}$ & $(2.19 \pm 0.04) \times 10^{-3}$ \\
\hline$[[23,1,7]]$ & 10 & 2 & 28,023 & $(4 \pm 1) \times 10^{-8}$ & $(3 \pm 2) \times 10^{-8}$ & $(5.76 \pm 0.09) \times 10^{-4}$ & $(5.48 \pm 0.09) \times 10^{-4}$ \\
\hline$[[23,1,7]]$ & 20 & 2 & 56,023 & $(5 \pm 1) \times 10^{-8}$ & $\approx<4 \times 10^{-8 d}$ & $(1.23 \pm 0.01) \times 10^{-3}$ & $(1.15 \pm 0.01) \times 10^{-3}$ \\
\hline$[[23,1,7]]$ & 30 & 2 & 84,023 & - & - & $(1.628 \pm 0.006) \times 10^{-3}$ & $(1.487 \pm 0.003) \times 10^{-3}$ \\
\hline$[[23,1,7]]$ & 40 & 2 & 112,023 & - & - & $(1.95 \pm 0.01) \times 10^{-3}$ & $(1.77 \pm 0.02) \times 10^{-3}$ \\
\hline
\end{tabular}

${ }^{a}$ for $[[5,1,3]]$ this parameter is NB.

${ }^{b}$ for $[[5,1,3]]$ this parameter is NC.

${ }^{c}$ for $[[5,1,3]]$ this parameter is the number of CNOT gates in a $T_{3}$ rectangle

${ }^{d}$ one failure in $5 \times 10^{7}$ samples

Table 3: Complete tabulation of code survey data, part 1 


\begin{tabular}{|c|c|c|c|c|c|c|c|}
\hline$[[n, k, d]]$ & $\mathrm{L}$ & $\mathrm{R}^{a}$ & CX/REC & $p_{1}\left(p_{\mathrm{mem}}=0, p_{0}=10^{-4}\right)$ & $p_{1}\left(p_{\text {mem }}=p_{0}=10^{-4}\right)$ & $p_{\mathrm{th}}\left(p_{\mathrm{mem}}=0\right)$ & $p_{\mathrm{th}}\left(p_{\mathrm{mem}}=p_{0}\right)$ \\
\hline$\overline{\overline{[[23,1,7]]}}$ & 10 & 3 & 40,023 & (4 $(4 \pm 2) \times 10^{-8}$ & 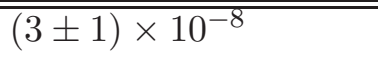 & 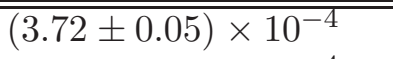 & $(3.45 \pm 0.05) \times 10^{-4}$ \\
\hline$[[23,1,7]]$ & 20 & 3 & 80,023 & - & & $(8.03 \pm 0.05) \times 10^{-4}$ & $(7.67 \pm 0.05) \times 10^{-4}$ \\
\hline$[[23,1,7]]$ & 30 & 3 & 120,023 & - & - & $(1.095 \pm 0.003) \times 10^{-3}$ & $(1.036 \pm 0.008) \times 10^{-3}$ \\
\hline$[[23,1,7]]$ & 40 & 3 & 160,023 & - & - & $(1.366 \pm 0.007) \times 10^{-3}$ & $(1.280 \pm 0.009) \times 10^{-3}$ \\
\hline$[[25,1,5]]$ & 4 & 1 & 1,465 & - & $(1.2 \pm 0.7) \times 10^{-6}$ & $(8.6 \pm 0.2) \times 10^{-4}$ & $(7.44 \pm 0.05) \times 10^{-4}$ \\
\hline$[[25,1,5]]$ & 5 & 1 & 1,825 & - & $(1.0 \pm 0.1) \times 10^{-6}$ & $(1.13 \pm 0.02) \times 10^{-3}$ & $(9.74 \pm 0.07) \times 10^{-4}$ \\
\hline$[[25,1,5]]$ & 6 & 1 & 2,185 & - & $(1.08 \pm 0.08) \times 10^{-6}$ & $(1.16 \pm 0.02) \times 10^{-3}$ & $(1.034 \pm 0.008) \times 10^{-3}$ \\
\hline$[[25,1,5]]$ & 7 & 1 & 2,545 & - & $(1.1 \pm 0.2) \times 10^{-6}$ & $(1.17 \pm 0.04) \times 10^{-3}$ & $(1.01 \pm 0.04) \times 10^{-3}$ \\
\hline$[[41,1,5]]$ & 5 & 1 & 11,321 & $(7.3 \pm 0.8) \times 10^{-6}$ & $(2.39 \pm 0.05) \times 10^{-4}$ & $(1.86 \pm 0.02) \times 10^{-4}$ & $(7.9 \pm 0.1) \times 10^{-5}$ \\
\hline$[[41,1,5]]$ & 10 & 1 & 22,601 & {$\left[3 \times 10^{-7}\right]^{b}$} & {$\left[7 \times 10^{-7}\right]$} & $(7.44 \pm 0.03) \times 10^{-4}$ & $(3.44 \pm 0.01) \times 10^{-4}$ \\
\hline$[[41,1,5]]$ & 15 & 1 & 33,881 & - & - & $(1.224 \pm 0.003) \times 10^{-3}$ & $(5.55 \pm 0.02) \times 10^{-4}$ \\
\hline$[[41,1,5]]$ & 20 & 1 & 45,161 & - & - & $(1.577 \pm 0.004) \times 10^{-3}$ & $(7.61 \pm 0.02) \times 10^{-4}$ \\
\hline$[[41,1,5]]$ & 30 & 1 & 67,721 & - & - & $(2.06 \pm 0.01) \times 10^{-3}$ & $(1.008 \pm 0.008) \times 10^{-3}$ \\
\hline$[[47,1,11]]$ & 10 & 1 & 52,527 & - & - & $(3.25 \pm 0.04) \times 10^{-4}$ & $(2.15 \pm 0.04) \times 10^{-4}$ \\
\hline$[[47,1,11]]$ & 20 & 1 & 105,007 & - & - & $(6.89 \pm 0.05) \times 10^{-4}$ & $(4.79 \pm 0.03) \times 10^{-4}$ \\
\hline$[[47,1,11]]$ & 30 & 1 & 157,487 & $(1.6 \pm 0.9) \times 10^{-7}$ & - & $(9.51 \pm 0.04) \times 10^{-4}$ & $(6.45 \pm 0.03) \times 10^{-4}$ \\
\hline$[[49,1,9]]$ & 5 & 2 & 61,549 & - & - & $(1.02 \pm 0.02) \times 10^{-4}$ & $(5.4 \pm 0.1) \times 10^{-5}$ \\
\hline$[[49,1,9]]$ & 10 & 2 & 123,049 & - & - & $(3.63 \pm 0.08) \times 10^{-4}$ & $(2.23 \pm 0.04) \times 10^{-4}$ \\
\hline$[[49,1,9]]$ & 15 & 2 & 184,549 & - & - & $(4.0 \pm 0.02) \times 10^{-4}$ & $(3.20 \pm 0.08) \times 10^{-4}$ \\
\hline$[[49,1,9]]$ & 20 & 2 & 246,049 & $(4 \pm 1) \times 10^{-6}$ & - & $(4.2 \pm 0.3) \times 10^{-4}$ & - \\
\hline$[[49,1,7]]$ & 4 & 1 & 2,961 & - & $(3.4 \pm 0.2) \times 10^{-6}$ & $(4.73 \pm 0.09) \times 10^{-4}$ & $(3.20 \pm 0.02) \times 10^{-4}$ \\
\hline$[[49,1,7]]$ & 6 & 1 & 4,417 & - & $(2.8 \pm 0.2) \times 10^{-7}$ & $(1.18 \pm 0.01) \times 10^{-3}$ & $(8.7 \pm 0.2) \times 10^{-4}$ \\
\hline$[[49,1,7]]$ & 8 & 1 & 5,873 & - & $(3.3 \pm 0.6) \times 10^{-7}$ & $(1.41 \pm 0.02) \times 10^{-3}$ & $(1.169 \pm 0.005) \times 10^{-3}$ \\
\hline$[[49,1,7]]$ & 9 & 1 & 6,601 & - & $(2.2 \pm 0.7) \times 10^{-7}$ & $(1.48 \pm 0.02) \times 10^{-3}$ & $(1.224 \pm 0.005) \times 10^{-3}$ \\
\hline$[[49,1,7]]$ & 10 & 1 & 7,329 & - & $(4.0 \pm 0.9) \times 10^{-7}$ & $(1.42 \pm 0.03) \times 10^{-3}$ & $(1.235 \pm 0.005) \times 10^{-3}$ \\
\hline$[[49,1,7]]$ & 11 & 1 & 8,057 & - & $(2.5 \pm 0.2) \times 10^{-7}$ & $(1.46 \pm 0.03) \times 10^{-3}$ & $(1.241 \pm 0.006) \times 10^{-3}$ \\
\hline$[[49,1,7]]$ & 12 & 1 & 8,785 & - & $(3 \pm 2) \times 10^{-7}$ & $(1.46 \pm 0.02) \times 10^{-3}$ & $(1.242 \pm 0.006) \times 10^{-3}$ \\
\hline$[[60,4,10]]$ & 10 & 1 & 86,460 & - & - & $(1.129 \pm 0.004) \times 10^{-4}$ & - \\
\hline$[[60,4,10]]$ & 20 & 1 & 172,860 & - & - & $(3.91 \pm 0.02) \times 10^{-4}$ & $(2.20 \pm 0.04) \times 10^{-4}$ \\
\hline
\end{tabular}

\footnotetext{
${ }^{a}$ for $[[49,1,9]]$ this parameter is the number of preparation attempts for a 7-qubit encoded ancilla used in error detection
}

${ }^{b}$ The values in square brackets are extrapolated from a linear least-squares fit to the logarithm of $p_{1}\left(p_{0}\right)$ 


\begin{tabular}{l|l|l|l|l|l|l|l}
{$[[n, k, d]]$} & $\mathrm{L}$ & $\mathrm{R}$ & $\mathrm{CX} /$ REC & $p_{1}\left(p_{\text {mem }}=0, p_{0}=10^{-4}\right)$ & $p_{1}\left(p_{\text {mem }}=p_{0}=10^{-4}\right)$ & $p_{\text {th }}\left(p_{\text {mem }}=0\right)$ & $p_{\text {th }}\left(p_{\text {mem }}=p_{0}\right)$ \\
\hline \hline$[[81,1,9]]$ & 4 & 1 & 4,977 & - & $(4.4 \pm 0.7) \times 10^{-5}$ & $(2.1 \pm 0.2) \times 10^{-4}$ & $(1.407 \pm 0.005) \times 10^{-4}$ \\
{$[[81,1,9]]$} & 6 & 1 & 7,425 & - & {$\left[1 \times 10^{-6}\right]^{a}$} & $(7.1 \pm 0.1) \times 10^{-4}$ & $(4.47 \pm 0.03) \times 10^{-4}$ \\
{$[[81,1,9]]$} & 10 & 1 & 12,321 & - & {$\left[7 \times 10^{-7}\right]$} & $(1.25 \pm 0.02) \times 10^{-3}$ & $(9.57 \pm 0.03) \times 10^{-4}$ \\
{$[[81,1,9]]$} & 11 & 1 & 13,545 & - & - & $(1.32 \pm 0.02) \times 10^{-3}$ & $(1.029 \pm 0.004) \times 10^{-3}$ \\
{$[[81,1,9]]$} & 12 & 1 & 14,769 & - & - & $(1.29 \pm 0.03) \times 10^{-3}$ & $(1.069 \pm 0.006) \times 10^{-3}$ \\
{$[[81,1,9]]$} & 18 & 1 & 22,113 & - & - & $(1.30 \pm 0.03) \times 10^{-3}$ & $(1.113 \pm 0.006) \times 10^{-3}$ \\
{$[[81,1,9]]$} & 19 & 1 & 23,337 & - & - & $(1.34 \pm 0.03) \times 10^{-3}$ & $(1.098 \pm 0.006) \times 10^{-3}$ \\
{$[[81,1,9]]$} & 20 & 1 & 24,561 & - & - & $(1.34 \pm 0.02) \times 10^{-3}$ & $(1.112 \pm 0.006) \times 10^{-3}$ \\
\hline$[[85,1,7]]$ & 5 & 1 & 30,405 & - & - & $(2.4 \pm \pm 0.1) \times 10^{-5}$ & $(2.03 \pm 0.07) \times 10^{-5}$ \\
{$[[85,1,7]]$} & 10 & 1 & 60,725 & - & - & $(4.18 \pm 0.05) \times 10^{-4}$ & $(1.03 \pm 0.04) \times 10^{-4}$ \\
{$[[85,1,7]]$} & 15 & 1 & 91,045 & - & - & $(5.59 \pm 0.04) \times 10^{-4}$ & $(1.76 \pm 0.02) \times 10^{-4}$ \\
{$[[85,1,7]]$} & 20 & 1 & 121,365 & - & {$\left[2 \times 10^{-7}\right]$} & $(2.32 \pm 0.02) \times 10^{-4}$
\end{tabular}

${ }^{a}$ The values in square brackets are extrapolated from a linear least-squares fit to the logarithm of $p_{1}\left(p_{0}\right)$

Table 5: Complete tabulation of code survey data, part 3 


\begin{tabular}{l|l|l|l}
{$[[n, k, d=\ell]]$} & CX/REC & $p_{1}\left(p_{\text {mem }}=p_{0}=10^{-4}\right)$ & $p_{\text {th }}\left(p_{\text {mem }}=p_{0}\right)$ \\
\hline \hline$[[41,1,5]]$ & 1,481 & $(1.7 \pm 0.1) \times 10^{-4}$ & $(6.8 \pm 0.6) \times 10^{-5}$ \\
{$[[85,1,7]]$} & 4,453 & $(5 \pm 2) \times 10^{-5}$ & $(2.3 \pm 0.2) \times 10^{-4}$ \\
{$[[145,1,9]]$} & 9,937 & $(2 \pm 1) \times 10^{-5}$ & $(4.5 \pm 0.2) \times 10^{-4}$ \\
{$[[221,1,11]]$} & 18,701 & {$\left[8 \times 10^{-6}\right]^{a}$} & $(6.6 \pm 0.2) \times 10^{-4}$ \\
{$[[313,1,13]]$} & 31,513 & {$\left[8 \times 10^{-6}\right]$} & $(9.0 \pm 0.4) \times 10^{-4}$
\end{tabular}

${ }^{a}$ The values in square brackets are extrapolated from a linear least-squares fit to the logarithm of $p_{1}\left(p_{0}\right)$

Table 6: Surface code data using Shor-EC and a transversal CNOT as in [12], taking $\ell$ syndromes for an $\ell \times \ell$ code EC. 


\begin{tabular}{l|l|l}
{$[[n, k, d]]$} & family & $p_{\text {th }}$ (perfect ancilla $)$ \\
\hline \hline$[[5,1,3]]$ & & $(2.0 \pm 0.1) \times 10^{-4}$ \\
{$[[7,1,3]]$} & doubly-even dual-containing & $(9.1 \pm 0.2) \times 10^{-4}$ \\
{$[[9,1,3]]$} & Bacon-Shor & $(6.0 \pm 0.9) \times 10^{-4}$ \\
{$[[13,1,3]]$} & surface & $(8.8 \pm 0.1) \times 10^{-4}$ \\
{$[[21,3,5]]$} & polynomial & $<10^{-5}$ \\
{$[[23,1,7]]$} & dual-containing & $(5.34 \pm 0.04) \times 10^{-3}$ \\
{$[[25,1,5]]$} & Bacon-Shor & $(1.88 \pm 0.04) \times 10^{-3}$ \\
{$[[41,1,5]]$} & surface & $(3.8 \pm 0.3) \times 10^{-3}$ \\
{$[[47,1,11]$} & doubly-even dual-containing & $(7.67 \pm 0.03) \times 10^{-3}$ \\
{$[[49,1,7]]$} & Bacon-Shor & $(2.56 \pm 0.05) \times 10^{-3}$ \\
{$[[49,1,9]]$} & doubly-even dual-containing & $(4.8 \pm 0.2) \times 10^{-3}$ \\
{$[[60,4,10]]$} & polynomial & $(1.88 \pm 0.04) \times 10^{-3}$ \\
{$[[81,1,9]]$} & Bacon-Shor & $(2.88 \pm 0.04) \times 10^{-3}$ \\
{$[[85,1,7]]$} & surface & $(2.5 \pm 0.3) \times 10^{-3}$ \\
{$[[121,1,11]]$} & Bacon-Shor & $(1.01 \pm 0.02) \times 10^{-2}$ \\
{$[[145,1,9]]$} & surface & $(2.97 \pm 0.09) \times 10^{-3}$ \\
{$[[169,1,13]]$} & Bacon-Shor &
\end{tabular}

Table 7: Level-1 pseudo-thresholds for rectangles using Steane-EC with perfect (noiseless) ancilla, $n<200$. 


\section{References}

[1] Panos Aliferis, Daniel Gottesman, and John Preskill. Quantum accuracy threshold for concatenated distance-3 codes. Quantum Information and Computation 6(2):97-165, 2006, http://arxiv.org/abs/quant-ph/0504218.

[2] K.M. Svore, B.M. Terhal, and D.P. DiVincenzo. Noise threshold for a fault-tolerant twodimensional lattice architecture. Quantum Information and Computation 7(4):297-318, 2007, http://arxiv.org/abs/quant-ph/0604090.

[3] Federico M. Spedalieri and Vwani P. Roychowdhury. Latency in local, two-dimensional, fault-tolerant quantum computing, 2008. http://arxiv.org/abs/0805.4213.

[4] Panos Aliferis and John Preskill. Fault-tolerant quantum computation against biased noise. Phys. Rev. A 78:052331, 2008, http://www.arxiv.org/abs/0710.1301.

[5] A. Steane. Overhead and noise threshold of fault-tolerant quantum error correction. Phys. Rev. A 68(4):42322-1-19, 2003, http://arxiv.org/abs/quant-ph/0207119.

[6] K.M. Svore, A. Cross, I.L. Chuang and A. Aho. A flow-map model for analyzing pseudothresholds in fault-tolerant quantum computing. Quantum Information and Computation 6(3):193-212. 2006, http://arxiv.org/abs/quant-ph/0508176

[7] M. Grassl, W. Geiselmann, and T. Beth. Quantum Reed-Solomon Codes. Proceedings Applied Algebra, Algebraic Algorithms and Error-Correcting Codes (AECC-13), Lecture Notes in Computer Science 1719, Springer, 1999, http: / / www .arxiv.org/abs/quant-ph/9910059.

[8] A. Steane. Space, time, parallelism and noise requirements for realiable quantum computing. Fortsch. Phys. 46:443-458, 1998, http://arxiv.org/abs/quant-ph/9708021.

[9] Panos Aliferis and John Preskill. The Fibonacci scheme for fault-tolerant quantum computation, 2008, http://www.arXiv.org/abs/0809.5063

[10] E. Knill. Quantum computing with realistically noisy devices. Nature 434:39-44, 2005.

[11] Robert Raussendorf, Jim Harrington, and Kovid Goyal. Topological fault-tolerance in cluster state quantum computation. New Journal of Physics 9:199, 2007.

[12] E. Dennis, A. Kitaev, A. Landahl, and J. Preskill. Topological quantum memory. J. Math. Phys. 43:4452-4505, 2002, http://arxiv.org/abs/quant-ph/0110143.

[13] A. Steane. Efficient fault-tolerant quantum computing. Nature 399:124-126, 1999, http://arxiv.org/abs/quant-ph/9809054.

[14] Ben W. Reichardt. Quantum universality by distilling certain one- and two-qubit states with stabilizer operations, 2006, http://arXiv.org/abs/quant-ph/0608085.

[15] S. Bravyi and A. Kitaev. Universal quantum computation with ideal Clifford gates and noisy ancillas. Phys. Rev. A 71:022316, 2005, http://arxiv.org/abs/quant-ph/0403025.

[16] A. Fowler. Towards large-scale quantum computation. PhD thesis, University of Melbourne, 2005, http://arxiv.org/abs/quant-ph/0506126.

[17] D. Gottesman. Stabilizer Codes and Quantum Error Correction. PhD thesis, CalTech, 1997, http://arxiv.org/abs/quant-ph/9705052. 
[19] P. Aliferis and A. Cross. Subsystem fault-tolerance with the Bacon-Shor code. Phys. Rev. Lett. 98:220502, 2007, http://www.arxiv.org/abs/quant-ph/0610063.

[20] D. Beckman, A. Chari, S. Devabhaktuni, and J. Preskill. Efficient networks for quantum factoring. Phys. Rev. A 54:1034-1063, 1996, http://www.arxiv.org/abs/quant-ph/9602016.

[21] R. Van Meter. Architecture of a quantum multicomputer optimized for Shor's factoring algorithm. Ph.D. thesis, Keio University, http://www.arxiv.org/abs/quant-ph/0607065.

[22] A. Steane. Quantum Reed-Muller codes. IEEE Trans. on Inf. Theory 45(5):1701-1703, 1999, http://arxiv.org/abs/quant-ph/9608026.

[23] E. Knill, R. Laflamme, and W. Zurek. Resilient quantum computation: Error models and thresholds. Proc. R. Soc. Lond. A 454:365-384, 1997, http://arxiv.org/abs/quant-ph/9702058.

[24] M. Grassl and T. Beth. Quantum BCH codes. In Proceedings X. International Symposium on Theoretical Electrical Engineering, pages 207-212, 1999, http://www.arxiv.org/abs/quant-ph/9910060.

[25] Ben W. Reichardt. Improved ancilla preparation scheme increases fault-tolerant threshold, 2004, http://arxiv.org/abs/quant-ph/0406025.

[26] D. Aharonov and M. Ben-Or. Fault-tolerant quantum computation with constant error rate. SIAM Journal of Computation 38(4):1207-1282, 2008, http://arxiv.org/abs/quant-ph/9906129.

[27] Raymond Laflamme, Cesar Miquel, Juan Pablo Paz, and Wojciech Hubert Zurek. Perfect quantum error correction code. Phys. Rev. Lett. 77:196-201,1996.

[28] S. B. Bravyi and A. Yu Kitaev. Quantum codes on a lattice with boundary, http://www.arXiv.org/abs/9811052.

[29] M. Freedman and D. Meyer. Projective plane and planar quantum codes. Found. of Comp. Math. 1:325-332, 2001, http://www.arxiv.org/abs/quant-ph/9810055.

[30] P. Aliferis. Level Reduction and the Quantum Threshold Theorem. PhD thesis, CalTech, 2007, http://arxiv.org/abs/quant-ph/0703230.

[31] P. Aliferis and J. Preskill. Private Communication.

[32] A. Steane. Fast fault-tolerant filtering of quantum codewords. 2002, http://arxiv.org/abs/quant-ph/0202036.

[33] A. Steane. Active stabilisation, quantum computation, and quantum state synthesis. Phys. Rev. Lett. 78:2252-2255, 1997, http://arxiv.org/abs/quant-ph/9608026.

[34] B. Reichardt. Error-detection-based quantum fault tolerance against discrete Pauli noise. $\mathrm{PhD}$ thesis, UC Berkeley, 2006, http://arxiv.org/abs/quant-ph/0612004.

[35] B. Zeng, A. Cross, and I. Chuang. Transversality versus universality for additive quantum codes. 2007, http://arxiv.org/abs/quant-ph/0706.1382.

[36] A. W. Cross, http://web.mit.edu/awcross/www/qasm-tools/. Software tools for synthesizing and evaluating fault-tolerant quantum gadgets.

[37] Wieb Bosma, John Cannon, and Catherine Playoust. The Magma algebra system. I. the user language. J. Symbolic Comput. 24(3-4):235-265, 1997. http: //magma .maths .usyd.edu.au/magma/. 
[39] Jasper Cramwinckel, Erik Roijackers, Reinald Baart, Eric Minkes, Lea Ruscio, Robert Miller, Tom Boothby, Cen Tjhai, and David Joyner. GAP package GUAVA. http://sage.math.washington.edu/home/wdj/guava/

[40] The message passing interface standard. http://www-unix.mcs.anl.gov/mpi/

[41] A. Partow. Galois field artithmetic library. http://www.partow.net/projects/galois/index.html.

[42] E. Rothberg. Implementation of Algorithms for Maximum Matching on Nonbipartite Graphs. PhD thesis, Stanford University, 1973. Implementation of H. Gabow's $O\left(n^{3}\right)$ weighted matching algorithm, ftp://dimacs.rutgers.edu/pub/netflow/matching/weighted/solver-1/.

[43] M. A. Nielsen and I.L. Chuang. Quantum Information and Quantum Computation. Cambridge University Press, 2000.

[44] Bryan Eastin. Fault-tolerant thresholds for encoded ancillae with homogeneous errors. Phys. Rev. A 75: 022301, 2007, http: //www.arxiv.org/abs/0605192.

[45] A. Steane and B. Ibinson. Fault-tolerant logical gate networks for CSS codes. Phys. Rev. A 72: 052335, 2005, http://arxiv.org/abs/quant-ph/0311014

[46] E.M. Rains. Quantum codes of minimum distance two. IEEE Trans. on Inf. Theory 45(1):266-271, 1999, http://arxiv.org/abs/quant-ph/9704043

[47] A. Kapoor and R. Rizzi. Edge-coloring bipartite graphs. J. Algorithms 34(2):390-396, 2000, http://citeseer.ist.psu.edu/726996.html.

[48] S. Aaronson and D. Gottesman. Improved simulation of stabilizer circuits. Phys. Rev. A 70:052328, 2004, http://arxiv.org/abs/quant-ph/0406196.

[49] Y.H. Chen, T.K. Truong, Y. Chang, C.D. Lee, and S.H. Chen. Algebraic decoding of quadratic residue codes using Berlekamp-Massey algorithm. Journal of Information Science and Engineering 23(1):127-145, 2007, http://www.iis.sinica.edu.tw/JISE/2007/200701_07.html

[50] W. Hufmann and V. Pless. Fundamentals of Error-Correcting Codes. Cambridge University Press, Cambridge, UK, 2003. 